J. Appl. Numer. Optim. 3 (2021), No. 2, pp. 333-359

Available online at http://jano.biemdas.com

https://doi.org/10.23952/jano.3.2021.2.07

\title{
A POSTERIORI ERROR CONTROL FOR VARIATIONAL INEQUALITIES WITH LINEAR CONSTRAINTS IN AN ABSTRACT FRAMEWORK
}

\author{
LOTHAR BANZ, ANDREAS SCHRÖDER* \\ Department of Mathematics, University of Salzburg, Hellbrunnerstrasse 34, 5020 Salzburg, Austria
}

\begin{abstract}
This paper proposes a posteriori error control for the discretization of variational inequalities with linear constraints in an abstract framework. The central aspect is the discussion of the error contributions representing the non-penetration, non-conformity and complementarity conditions, which are typically given by some cut-off functions. Replacing the standard cut-off functions with the minimizers of a weighted functional enables the derivation of reliable and, in particular, efficient a posteriori error estimates. The abstract findings are applied to the obstacle problem as well as to the simplified Signorini problem, where higher-order finite elements are used to provide appropriate discretization spaces. Numerical experiments show that the error estimates based on this new approach have (nearly) constant efficiency indeces and reflect the expected order of convergence when uniform $h$-refinements are applied. Moreover, they can be used to steer adaptive schemes in order to improve the order of convergence. The numerical results are compared with estimates resulting from the standard cut-off functions.
\end{abstract}

Keywords. Variational inequality; A poseriori error control; Higher-order finite elements.

\section{INTRODUCTION}

Variational inequalities are an important tool for the mathematical modeling of problems in mechanical engineering, in financial mathematics and in many other application areas. They are used to model contact problems [1, 2, 3, 4], to characterize elastoplastic material behavior [5] or to describe financial options [6]. In many cases (e.g. in the modeling of contact problems) they are given by an elliptic bilinear form together with linear constraints which, for instance, describe non-penetration conditions of the solution or its gradient. In order to discretize variational inequalities finite elements are usually applied. The control of the discretization error in terms of the discretization solution is essential to measure the quality of the discretization and, moreover, to steer adaptive schemes. This so-called a posteriori error control requires the derivation of upper and lower bounds leading to reliable and efficent a posteriori error estimates, respectively, $[7,8]$. In the literature several approaches of a posteriori error control are proposed in the context of the discretization of variational inequalities. They are often transferred from error control approaches for variational equations and are based, for instance, on averaging techniques, e.g., [9], or residual-based approaches, e.g., [10, 11, 12, 13, 14, 15, 16],

\footnotetext{
${ }^{*}$ Corresponding author.
}

E-mail addresses: lothar.banz@sbg.ac.at (L. Banz), andreas.schroeder@sbg.ac.at (A. Schröder).

Received December 28, 2020; Accepted April 6, 2021.

(C)2021 Journal of Applied and Numerical Optimization 
the hypercircle method [17], $H($ div)-conforming stress approximations [16] or dual weighted residual approaches $[18,19,20]$.

To derive a posteriori error estimates, one can use the fact that the discretization solution (or an arbitrary function of the discretization space) defines a certain residuum which is associated with the constraints and which can be interpreted as a Lagrange multliplier. It specifies an auxiliary problem which is just a variational equation and whose discretization is also solved by the discretization solution (or by the arbitrary function of the discretization space). In [21], this fundamental approach has been presented for the derivation of error estimates for the obstacle problem. In particular, it is shown that the discretization error of the obstacle problem can be bounded by the error resulting from the discretization of the auxiliary problem and by some additional terms representing non-penetration and complementarity conditions. Standard techniques to control the discretization error for variational equations can then be applied to estimate the discretization error of the auxiliary problem which is given by the Poisson equation in the case of the (standard) obstacle problem.

In this paper, a posteriori error control for the discretization of variational inequalities with linear constraints is proposed in an abstract framework. It extends the approaches introduced, for instance, in $[22,23,24,25,26]$ to a setting which is formulated in real Hilbert spaces and which is, in particular, appropriate for discretizations with higher-order finite elements. The central aspect of this paper consists in the discussion of the error contributions representing the non-penetration, non-conformity and complementarity conditions, which are typically expressed by using some cut-off functions. In the previous works [24, 25, 26, 27, 28], it was shown that the standard cut-off function $\max \{f, 0\}$ for an $L^{2}$-function $f$ gives reliable estimates, in particular, for higher-order and $h p$-adaptive finite elements. In several numerical experiments, it is demonstrated that these estimates also seem to be efficient. Unfortunately, it is not clear whether the use of the standard cut-off functions gives efficiency also in theory. To overcome this problem, we propose a new approach that replaces the standard cut-off functions by the minimizers of a weighted functional resulting from the non-penetration, the non-conformity and the complementarity conditions. These minimizers can explicitly be determined by using general positive parts or, more precisely, appropriately approximated by some finite element cut-off functions and lead to reliable and, in particular, efficient a posteriori error estimates. Furthermore, they are strongly connected to the Lagrange multiplier and the nonpenetration condition. We apply the abstract findings to the obstacle problem as well as to the simplified Signorini problem, where we use higher-order finite elements to provide suitable discretization spaces. We point out that this approach can also be applied to related variational inequalities with linear constraints as, for instance, problems in linear elasticity. Indeed, in $[25,27,28,29,30,31,32]$ similar techniques have already been applied for the derivation of reliable a posteriori error estimates for various linear elastic contact problems discretized by finite elements or boundary element methods. These approaches can be straightforwardly extended by using the abstract findings of this paper. In numerical experiments we show that the error estimates based on the finite element cut-off functions have (nearly) constant efficiency indeces and reflect the expected order of convergence when uniform $h$-refinements and higher-order finite elements are applied. Moreover, they can be used to steer adaptive schemes in order to improve the order of convergence, which is, however, limited due to the reduced regularity of the solution at the curved interface separating contact from non-contact and due to the use of 
isotropic refinements. We compare the results with estimates resulting from the standard cutoff functions and observe that these results only slightly differ, which possibly implies that the standard cut-off functions and the finite element cut-off functions are strongly related in some sense.

The paper is organized as follows. In Section 2, we introduce the abstract framework in which the error control for variational inequalities with linear constraints is presented in real Hilbert spaces. A special emphasis lies on the introduction of the Lagrange multiplier associated with the linear constraints. We derive error estimates including a weighted functional and, alternatively a non-weighted functional, which, however, prevents the derivation of efficient estimates. Within this abstract framework we discuss the minimizers of the weighted functional, their approximations and their relation to the Lagrange multiplier and the non-penetration condition. In Section 3, we introduce the obstacle problem and the simplified Signorini problem as model problems for variational inequalities with linear constraints and discuss some higher-order finite element discretization spaces. Furthermore, we assemble all ingredients, which are necessary for the derivation of the a posteriori error estimates based on the abstract framework. In particular, we present the residual error estimator from [33] in this section, which is appropriate for higher-order finite elements. Eventually, the application of the abstract estimates gives reliable and efficient a posteriori error estimates for the obstacle problem as well as for the simplified Signorini problem. We note that the error estimates are applicable for an arbitrary function of the discretization space and are not limited to discretization solutions of these model problems. Section 4 contains the numerical experiments and, in particular, the comparison of error estimates resulting from the standard cut-off functions and the finite element cut-off functions.

\section{THE ABSTRACT FRAMEWORK}

2.1. Variational inequalities with linear constraints. Let $Z$ be a real Hilbert space and let $\ell \in Z^{*}$ and $B: Z \rightarrow Z^{*}$ be a linear, continuous and $Z$-coercive mapping, i.e.,

$$
\|B z\|_{Z^{*}} \leq c\|z\|_{Z}, \quad\langle B z, z\rangle \geq \beta\|z\|_{Z}^{2}
$$

for all $z \in Z$ and some constants $c, \beta>0$, where $\langle\cdot, \cdot\rangle$ denotes the dual pairing of $Z$ to $Z^{*}$. For a further real Hilbert space $Y$, let $G \subset Y$ be a closed and convex cone with $0 \in G$ and $G^{*}:=\{\mu \in$ $\left.Y^{*} \mid \forall w \in G:\langle\mu, w\rangle \geq 0\right\}$ be its dual cone. Furthermore, let $\gamma: Z \rightarrow Y$ be a linear, continuous and surjective operator with $\|\gamma(z)\|_{Y} \leq c_{\gamma}\|z\|_{Z}$ and define $K:=\{z \in Z \mid \gamma(z)-\psi \in G\}$ for some $\psi \in Y$. We consider the variational inequality: Find $u \in K$ such that

$$
\langle B u-\ell, z-u\rangle \geq 0
$$

for all $z \in K$.

Theorem 2.1. The variational inequality (2.1) has a unique solution, which Lipschitz-continuously depends on the data $\ell$.

Proof. See, e.g., [34, Th. 2.1].

Lemma 2.1. Let $\mu: Y \rightarrow \mathbb{R}$ be linear. Then, $\mu \in Y^{*}$ if and only if $\mu \circ \gamma \in Z^{*}$. Moreover, there exists a constant $\hat{c}_{\gamma}>0$ such that

$$
c_{\gamma}^{-1}\|\mu \circ \gamma\|_{Z^{*}} \leq\|\mu\|_{Y^{*}} \leq \hat{c}_{\gamma}\|\mu \circ \gamma\|_{Z^{*}}
$$


Proof. If $\mu \in Y^{*}$, then

$$
\sup _{z \in Z \backslash\{0\}} \frac{\langle\mu \circ \gamma, z\rangle}{\|z\|_{Z}}=\sup _{z \in Z \backslash\{0\}} \frac{\langle\mu, \gamma(z)\rangle}{\|z\|_{Z}} \leq c_{\gamma}\|\mu\|_{Y^{*}},
$$

which shows $\mu \circ \gamma \in Z^{*}$ and the first inequality in (2.2). Let $\mu \circ \gamma \in Z^{*}$. The isomorphism theorem and the surjectivity of $\gamma$ imply a continuous isomorphism $\hat{\gamma}: Z / \operatorname{ker} \gamma \rightarrow Y$ with $\gamma=\hat{\gamma} \circ \phi$ and $\left\|\hat{\gamma}^{-1}(y)\right\|_{Z / \operatorname{ker} \gamma} \leq \hat{c}_{\gamma}\|y\|_{Y}$ for all $y \in Y$, where $\phi: Z \rightarrow Z /$ ker $\gamma$ denotes the natural projection. Hence,

$$
\begin{aligned}
\sup _{y \in Y \backslash\{0\}} \frac{\langle\mu, y\rangle}{\|y\|_{Y}} & =\sup _{z \in Z \backslash\{0\}} \inf _{v \in \operatorname{ker} \gamma} \frac{\langle\mu \circ \gamma, z+v\rangle}{\|\gamma(z)\|_{Y}} \leq\|\mu \circ \gamma\|_{Z^{*}} \sup _{z \in Z \backslash\{0\}} \inf _{v \in \operatorname{ker} \gamma} \frac{\|z+v\|_{Z}}{\|\gamma(z)\|_{Y}} \\
& \leq\|\mu \circ \gamma\|_{Z^{*}} \sup _{z \in Z \backslash\{0\}} \frac{\|\phi(z)\|_{Z / \operatorname{ker} \gamma}}{\|\gamma(z)\|_{Y}} \leq\|\mu \circ \gamma\|_{Z^{*}} \sup _{z \in Z \backslash\{0\}} \frac{\left\|\hat{\gamma}^{-1}(\gamma(z))\right\|_{Z / \operatorname{ker} \gamma}}{\|\gamma(z)\|_{Y}} \\
& \leq \hat{c}_{\gamma}\|\mu \circ \gamma\|_{Z^{*}},
\end{aligned}
$$

which implies $\mu \in Y^{*}$ and is the second inequality in (2.2).

Theorem 2.2. There exists a unique $\lambda \in G^{*}$ such that

$$
B u-\lambda \circ \gamma=\ell \text {. }
$$

Furthermore, it holds $\langle\lambda, \gamma(u)-\psi\rangle=0$ and

$$
\langle\mu-\lambda, \gamma(u)-\psi\rangle \geq 0
$$

for all $\mu \in G^{*}$.

Proof. Since $\gamma(u \pm z)-\psi=\gamma(u)-\psi \in G$ for $z \in \operatorname{ker} \gamma$, we obtain from inserting $u \pm z$ in (2.1) that $\langle B u-\ell, z\rangle=0$ and, thus, $\operatorname{ker} \gamma \subset \operatorname{ker}(B u-\ell)$. Hence, for $y \in Y$ and $z, \tilde{z} \in Z$ with $y=\gamma(z)=\gamma(\tilde{z})$ (note that $\gamma$ is surjective) we conclude $z-\tilde{z} \in \operatorname{ker}(B u-\ell$ ), which means that the linear functional $\lambda: Y \rightarrow \mathbb{R}$ with $\lambda(y):=\langle B u-\ell, z\rangle$ is well-defined. Obviously, $\lambda$ fulfills (2.3) and does this uniquely due to the surjectivity of $\gamma$. Lemma 2.1 and (2.3) imply $\lambda \in Y^{*}$. For $z \in Z$ with $\gamma(z) \in G$, we conclude from the convex cone property of $G$ that

$$
\gamma(u+z)-\psi=2\left(\frac{1}{2}(\gamma(u)-\psi)+\frac{1}{2} \gamma(z)\right) \in G
$$

and, therefore, $u+z \in K$. Due to (2.1), we get $\langle\lambda, \gamma(z)\rangle=\langle B u-\ell,(z+u)-u\rangle \geq 0$, which is $\lambda \in G^{*}$, where the surjectivity of $\gamma$ is exploited again. Since $\gamma(u)-\psi \in G$, we have $\langle\lambda, \gamma(u)-$ $\psi\rangle \geq 0$. From $0 \in G$ and (2.1), we conclude

$$
\langle\lambda, \psi-\gamma(u)\rangle=\langle B u-\ell, w-u\rangle \geq 0
$$

for some $w \in K$ with $\gamma(w)=\psi$. This, eventually, gives $\langle\lambda, \gamma(u)-\psi\rangle=0$. Since $\gamma(u)-\psi \in G$, we have

$$
\langle\mu-\lambda, \gamma(u)-\psi\rangle=\langle\mu, \gamma(u)-\psi\rangle \geq 0
$$

for all $\mu \in G^{*}$, which is (2.4).

Corollary 2.1. The pair $(u, \lambda) \in Z \times G^{*}$ uniquely fulfills the system (2.3) and (2.4). 
Proof. Applying Theorem 2.2, we only have to show the uniqueness of the first component. To this end, let $(\bar{u}, \lambda) \in Z \times G^{*}$ fulfill the system (2.3) and (2.4). Since $0,2 \lambda \in G^{*}$,we have from (2.4) that $\langle\lambda, \gamma(\bar{u})-\psi\rangle=0$. Thus, for $z \in K$, we have

$$
\langle B \bar{u}-\ell, z-\bar{u}\rangle=\langle\lambda, \gamma(z-\bar{u})\rangle=\langle\lambda, \gamma(z)-\psi\rangle+\langle\lambda, \psi-\gamma(\bar{u})\rangle \geq 0 .
$$

Since $u \in K$ uniquely solves (2.1), we have $\bar{u}=u$.

2.2. A posteriori error control. Let $v \in Z$ and $\kappa \in Y^{*}$ be arbitrarily chosen. Furthermore, define $K_{v}:=\{\zeta \in Y \mid \zeta+\gamma(v)-\psi \in G\}$ and the weighted functional

$$
\Xi_{\varepsilon}(\mu, \zeta):=\|\mu-\kappa\|_{Y^{*}}^{2}+\|\zeta\|_{Y}^{2}+\varepsilon\langle\kappa, \zeta\rangle+\varepsilon\langle\mu, \gamma(v)-\psi\rangle
$$

for $\mu \in Y^{*}, \zeta \in Y$ and $\varepsilon>0$.

Theorem 2.3. Let $0<\delta<\beta$ and $0<\varepsilon<\theta^{-1}$ and

$$
E:=\left(\frac{1+2 c^{2} \hat{c}_{\gamma}^{2}}{4 \delta(\beta-\delta)}+2 \hat{c}_{\gamma}^{2}\right), \quad F:=\frac{1+2 c^{2} \hat{c}_{\gamma}^{2}}{\beta-\delta}, \quad \theta:=\frac{F \max \left\{1, c_{\gamma}^{2}\right\}}{4}
$$

Then,

$$
\|u-v\|_{Z}^{2}+\|\lambda-\kappa\|_{Y^{*}}^{2} \leq \frac{E}{1-\theta \varepsilon}\|B v-\ell-\kappa \circ \gamma\|_{Z^{*}}^{2}+\frac{F}{\varepsilon(1-\theta \varepsilon)} \Xi_{\varepsilon}(\mu, \zeta)
$$

for all $\mu \in G^{*}$ and $\zeta \in K_{v}$.

Proof. Using (2.3) and Young's inequality, we conclude

$$
\begin{aligned}
\beta\|u-v\|_{Z}^{2} & \leq\langle B(u-v), u-v\rangle \\
& =\langle\ell+\lambda \circ \gamma-B v, u-v\rangle \\
& =\langle\ell+\kappa \circ \gamma-B v, u-v\rangle+\langle\lambda-\kappa, \gamma(u-v)\rangle \\
& \leq \frac{1}{4 \delta}\|B v-\ell-\kappa \circ \gamma\|_{Z^{*}}^{2}+\delta\|u-v\|_{Z}^{2}+\langle\lambda-\kappa, \gamma(u-v)\rangle,
\end{aligned}
$$

which gives

$$
\|u-v\|_{Z}^{2} \leq \frac{1}{4 \delta(\beta-\delta)}\|B v-\ell-\kappa \circ \gamma\|_{Z^{*}}^{2}+\frac{1}{\beta-\delta}\langle\lambda-\kappa, \gamma(u-v)\rangle
$$

for some $0<\delta<\beta$. Moreover, Lemma 2.1 and Theorem 2.2 imply

$$
\begin{aligned}
\|\lambda-\kappa\|_{Y^{*}} & \leq \hat{c}_{\gamma}\|(\lambda-\kappa) \circ \gamma\|_{Z^{*}} \\
& =\hat{c}_{\gamma}\|B u-\ell-\kappa \circ \gamma\|_{Z^{*}} \\
& \leq \hat{c}_{\gamma}\left(\|B(u-v)\|_{Z^{*}}+\|B v-\ell-\kappa \circ \gamma\|_{Z^{*}}\right) .
\end{aligned}
$$

Hence,

$$
\begin{aligned}
\|u-v\|_{Z}^{2}+\|\lambda-\kappa\|_{Y^{*}}^{2} & \leq\|u-v\|_{Z}^{2}+2 \hat{c}_{\gamma}^{2}\|B(u-v)\|_{Z^{*}}^{2}+2 \hat{c}_{\gamma}^{2}\|B v-\ell-\kappa \circ \gamma\|_{Z^{*}}^{2} \\
& \leq\left(1+2 c^{2} \hat{c}_{\gamma}^{2}\right)\|u-v\|_{Z}^{2}+2 \hat{c}_{\gamma}^{2}\|B v-\ell-\kappa \circ \gamma\|_{Z^{*}}^{2} \\
& \leq E\|B v-\ell-\kappa \circ \gamma\|_{Z^{*}}^{2}+F\langle\lambda-\kappa, \gamma(u-v)\rangle .
\end{aligned}
$$


From Theorem 2.2, we obtain

$$
\begin{aligned}
\langle\lambda & -\kappa, \gamma(u-v)\rangle \\
& =\langle\mu-\kappa, \gamma(u-v)\rangle+\langle\lambda-\mu, \gamma(u)-\psi\rangle+\langle\lambda-\mu, \psi-\gamma(v)\rangle \\
& \leq\langle\mu-\kappa, \gamma(u-v)\rangle+\langle\lambda-\mu, \psi-\gamma(v)\rangle \\
& =\langle\mu-\kappa, \gamma(u-v)\rangle+\langle\lambda, \psi-\gamma(v)-\zeta\rangle+\langle\lambda-\kappa, \zeta\rangle+\langle\kappa, \zeta\rangle+\langle\mu, \gamma(v)-\psi\rangle \\
& \leq \frac{\max \left\{1, c_{\gamma}^{2}\right\} \varepsilon}{4}\left(\|u-v\|_{Z}^{2}+\|\lambda-\kappa\|_{Y^{*}}^{2}\right)+\frac{1}{\varepsilon}\left(\|\mu-\kappa\|_{Y^{*}}^{2}+\|\zeta\|_{Y}^{2}\right)+\langle\kappa, \zeta\rangle+\langle\mu, \gamma(v)-\psi\rangle .
\end{aligned}
$$

Thus, we have

$$
\|u-v\|_{Z}^{2}+\|\lambda-\kappa\|_{Y^{*}}^{2} \leq E\|B v-\ell-\kappa \circ \gamma\|_{Z^{*}}^{2}+\theta \varepsilon\left(\|u-v\|_{Z}^{2}+\|\lambda-\kappa\|_{Y^{*}}^{2}\right)+\frac{F}{\varepsilon} \Xi_{\varepsilon}(\mu, \zeta) .
$$

Subtracting $\theta \varepsilon\left(\|u-v\|_{Z}^{2}+\|\lambda-\kappa\|_{Y^{*}}^{2}\right)$ and dividing by $1-\theta \varepsilon>0$ give the assertion.

The basic idea for the derivation of a posteriori error estimates is to transfer error estimates for variational equations to variational inequalities. For this purpose, we consider the solution $\hat{v} \in Z$ of the auxiliary problem

$$
B \hat{v}=\ell+\kappa \circ \gamma
$$

Note that the unique existence of $\hat{v}$ is guaranteed by the Lax-Milgram lemma. Furthermore, let $\hat{\eta} \geq 0$ such that

$$
\begin{aligned}
\hat{C} \hat{\eta}^{2} & \leq\|\hat{v}-v\|_{Z}^{2}+\mathrm{osc}^{2}, \\
\|\hat{v}-v\|_{Z}^{2} & \leq \hat{D}\left(\hat{\eta}^{2}+\mathrm{osc}^{2}\right)
\end{aligned}
$$

with some constants $\hat{C}, \hat{D}>0$ and some (data oscillations) osc $\geq 0$.

Lemma 2.2. It holds

$$
\frac{1}{c}\|B v-\ell-\kappa \circ \gamma\|_{Z^{*}} \leq\|\hat{v}-v\|_{Z} \leq \frac{c}{\beta}\|u-v\|_{Z}+\frac{c_{\gamma}}{\beta}\|\lambda-\kappa\|_{Y^{*}} .
$$

Proof. We have

$$
\|B v-\ell-\kappa \circ \gamma\|_{Z^{*}}=\sup _{z \in Z \backslash\{0\}} \frac{\langle B v-\ell-\kappa \circ \gamma, z\rangle}{\|z\|_{Z}}=\sup _{z \in Z \backslash\{0\}} \frac{\langle B(v-\hat{v}), z\rangle}{\|z\|_{Z}} \leq c\|\hat{v}-v\|_{Z},
$$

which gives the first inequality. The second inequality follows from

$$
\begin{aligned}
\beta\|\hat{v}-v\|_{Z}^{2} & \leq\langle B(\hat{v}-v), \hat{v}-v\rangle \\
& =\langle\kappa-\lambda, \gamma(\hat{v}-v)\rangle+\langle B(u-v), \hat{v}-v\rangle \\
& \leq\left(c_{\gamma}\|\lambda-\kappa\|_{Y^{*}}+c\|u-v\|_{Z}\right)\|\hat{v}-v\|_{Z} .
\end{aligned}
$$

Theorem 2.4. Let $0<\varepsilon<\min \left\{F\left(E c^{2} \hat{D}\right)^{-1}, \theta^{-1}\right\}$ and

$$
C:=\max \left\{\frac{2 c^{2} \hat{C}^{-1}}{\beta^{2}}+2 c_{\gamma}^{2}, \frac{2 c_{\gamma}^{2} \hat{C}^{-1}}{\beta^{2}}+1+\frac{\varepsilon^{2}}{4}, \hat{C}^{-1}\right\}^{-1}, \quad D:=\frac{F}{\varepsilon(1-\theta \varepsilon)} .
$$

Then,

$$
C\left(\hat{\eta}^{2}+\Xi_{\varepsilon}(\mu, \zeta)\right) \leq\|u-v\|_{Z}^{2}+\|\lambda-\kappa\|_{Y^{*}}^{2}+\operatorname{osc}^{2}
$$


for all $\mu \in Y^{*}$ and $\zeta \in Y$ with

$$
\Xi_{\varepsilon}(\mu, \zeta) \leq \Xi_{\varepsilon}(\lambda, \gamma(u-v))
$$

Furthermore, it holds

$$
\|u-v\|_{Z}^{2}+\|\lambda-\kappa\|_{Y^{*}}^{2} \leq D\left(\hat{\eta}^{2}+\Xi_{\varepsilon}(\mu, \zeta)+\operatorname{osc}^{2}\right)
$$

for all $\mu \in G^{*}$ and $\zeta \in K_{v}$.

Proof. From $\langle\lambda, \gamma(u)\rangle=\langle\lambda, \psi\rangle$, we conclude

$$
\begin{aligned}
\Xi_{\varepsilon}(\lambda, \gamma(u-v)) & =\|\lambda-\kappa\|_{Y^{*}}^{2}+\|\gamma(u-v)\|_{Y}^{2}+\varepsilon\langle\lambda, \gamma(v)-\psi\rangle+\varepsilon\langle\kappa, \gamma(u-v)\rangle \\
& =\|\lambda-\kappa\|_{Y^{*}}^{2}+\|\gamma(u-v)\|_{Y}^{2}+\varepsilon\langle\lambda-\kappa, \gamma(v-u)\rangle \\
& \leq\|\lambda-\kappa\|_{Y^{*}}^{2}+\|\gamma(u-v)\|_{Y}^{2}+\varepsilon\|\lambda-\kappa\|_{Y^{*}}\|\gamma(v-u)\|_{Y} \\
& \leq\left(1+\frac{\varepsilon^{2}}{4}\right)\|\lambda-\kappa\|_{Y^{*}}^{2}+2 c_{\gamma}^{2}\|u-v\|_{Z}^{2} .
\end{aligned}
$$

Thus, applying Lemma 2.2, we obtain for $\mu \in Y^{*}$ and $\zeta \in Y$ with $\Xi_{\varepsilon}(\mu, \zeta) \leq \Xi_{\varepsilon}(\lambda, \gamma(u-v))$

$$
\begin{aligned}
\hat{\eta}^{2}+\Xi_{\varepsilon}(\mu, \zeta) & \leq \hat{C}^{-1}\left(\|\hat{v}-v\|_{Z}^{2}+\operatorname{osc}^{2}\right)+\left(1+\frac{\varepsilon^{2}}{4}\right)\|\lambda-\kappa\|_{Y^{*}}^{2}+2 c_{\gamma}^{2}\|u-v\|_{Z}^{2} \\
& \leq\left(\frac{2 c^{2} \hat{C}^{-1}}{\beta^{2}}+2 c_{\gamma}^{2}\right)\|u-v\|_{Z}^{2}+\left(\frac{2 c_{\gamma}^{2} \hat{C}^{-1}}{\beta^{2}}+1+\frac{\varepsilon^{2}}{4}\right)\|\lambda-\kappa\|_{Y^{*}}+\hat{C}^{-1} \mathrm{osc}^{2},
\end{aligned}
$$

which gives the lower bound (2.8). Applying Theorem 2.3 and Lemma 2.2, we get for $\mu \in G^{*}$ and $\zeta \in K_{v}$

$$
\begin{aligned}
\|u-v\|_{Z}^{2}+\|\lambda-\kappa\|_{Y^{*}}^{2} & \leq \frac{E c^{2}}{1-\theta \varepsilon}\|\hat{v}-v\|_{Z}^{2}+\frac{F}{\varepsilon(1-\theta \varepsilon)} \Xi_{\varepsilon}(\mu, \zeta) \\
& \leq \frac{E c^{2} \hat{D}}{1-\theta \varepsilon}\left(\hat{\eta}^{2}+\operatorname{osc}^{2}\right)+\frac{F}{\varepsilon(1-\theta \varepsilon)} \Xi_{\varepsilon}(\mu, \zeta) \\
& \leq \frac{F}{\varepsilon(1-\theta \varepsilon)}\left(\hat{\eta}^{2}+\Xi_{\varepsilon}(\mu, \zeta)+\operatorname{osc}^{2}\right)
\end{aligned}
$$

due to the assumption on $\varepsilon$. This is the upper bound (2.10).

Alternatively, we may use the non-weighted functional

$$
\Xi(\mu, \zeta):=\|\mu-\kappa\|_{Y^{*}}^{2}+\|\zeta\|_{Y}^{2}+|\langle\mu, \gamma(v)-\psi\rangle+\langle\kappa, \zeta\rangle|
$$

for $\mu \in Y^{*}$ and $\zeta \in Y$ instead of $\Xi_{\varepsilon}(\mu, \zeta)$.

Theorem 2.5. Let $0<\varepsilon<\min \left\{\theta^{-1}, 1\right\}$ and

$$
\tilde{C}:=\max \left\{\frac{2 c^{2} \hat{C}^{-1}}{\beta^{2}}+2 c_{\gamma}^{2}, \frac{2 c_{\gamma}^{2} \hat{C}^{-1}}{\beta^{2}}+\frac{5}{4}, \hat{C}^{-1}\right\}^{-1} \quad, \quad \tilde{D}:=\max \left\{\frac{E c^{2} \hat{D}}{1-\theta \varepsilon}, \frac{F}{\varepsilon(1-\theta \varepsilon)}\right\} .
$$

Then,

$$
\tilde{C}\left(\hat{\eta}^{2}+\Xi(\mu, \zeta)\right) \leq\|u-v\|_{Z}^{2}+\|\lambda-\kappa\|_{Y^{*}}^{2}+\mathrm{osc}^{2}
$$

for all $\mu \in Y^{*}$ and $\zeta \in Y$ with

$$
\Xi(\mu, \zeta) \leq \Xi(\lambda, \gamma(u-v))
$$


Furthermore, it holds

$$
\|u-v\|_{Z}^{2}+\|\lambda-\kappa\|_{Y^{*}}^{2} \leq \tilde{D}\left(\hat{\eta}^{2}+\Xi(\mu, \zeta)+\operatorname{osc}^{2}\right)
$$

for all $\mu \in G^{*}$ and $\zeta \in K_{v}$.

Proof. We conclude from $\langle\lambda, \gamma(u)\rangle=\langle\lambda, \psi\rangle$ that

$$
\begin{aligned}
\Xi(\lambda, \gamma(u-v)) & =\|\lambda-\kappa\|_{Y^{*}}^{2}+\|\gamma(u-v)\|_{Y}^{2}+|\langle\lambda, \gamma(v)-\psi\rangle+\langle\kappa, \gamma(u-v)\rangle| \\
& =\|\lambda-\kappa\|_{Y^{*}}^{2}+\|\gamma(u-v)\|_{Y}^{2}+|\langle\lambda-\kappa, \gamma(v-u)\rangle| \\
& \leq\|\lambda-\kappa\|_{Y^{*}}^{2}+\|\gamma(u-v)\|_{Y}^{2}+\|\lambda-\kappa\|_{Y^{*}}\|\gamma(v-u)\|_{Y} \\
& \leq \frac{5}{4}\|\lambda-\kappa\|_{Y^{*}}^{2}+2 c_{\gamma}^{2}\|u-v\|_{Z}^{2} .
\end{aligned}
$$

Thus, applying Lemma 2.2 we obtain for all $\mu \in Y^{*}$ and $\zeta \in Y$ with $\Xi(\mu, \zeta) \leq \Xi(\lambda, \gamma(u-v))$

$$
\begin{aligned}
\hat{\eta}^{2}+\Xi(\mu, \zeta) & \leq \hat{C}^{-1}\left(\|\hat{v}-v\|_{Z}^{2}+\mathrm{osc}^{2}\right)+\frac{5}{4}\|\lambda-\kappa\|_{Y^{*}}^{2}+2 c_{\gamma}^{2}\|u-v\|_{Z}^{2} \\
& \leq\left(\frac{2 c^{2} \hat{C}^{-1}}{\beta^{2}}+2 c_{\gamma}^{2}\right)\|u-v\|_{Z}^{2}+\left(\frac{2 c_{\gamma}^{2} \hat{C}^{-1}}{\beta^{2}}+\frac{5}{4}\right)\|\lambda-\kappa\|_{Y^{*}}+\hat{C}^{-1} \mathrm{osc}^{2},
\end{aligned}
$$

which gives the lower bound (2.11). Note that

$$
\Xi_{\varepsilon}(\mu, \zeta) \leq \Xi(\mu, \zeta)
$$

for all $\mu \in G^{*}$ and $\zeta \in K_{v}$ due to the assumption $\varepsilon \leq 1$. Thus, from Theorem 2.3 and Lemma 2.2, we get for all $\mu \in G^{*}$ and $\zeta \in K_{v}$

$$
\begin{aligned}
\|u-v\|_{Z}^{2}+\|\lambda-\kappa\|_{Y^{*}}^{2} & \leq \frac{E c^{2}}{1-\theta \varepsilon}\|\hat{v}-v\|_{Z}^{2}+\frac{F}{\varepsilon(1-\theta \varepsilon)} \Xi_{\varepsilon}(\mu, \zeta) \\
& \leq \frac{E c^{2} \hat{D}}{1-\theta \varepsilon}\left(\hat{\eta}^{2}+\mathrm{osc}^{2}\right)+\frac{F}{\varepsilon(1-\theta \varepsilon)} \Xi(\mu, \zeta),
\end{aligned}
$$

which gives the upper bound (2.13).

Remark 2.1. The availability of $\hat{\eta}$ fulfilling (2.6) and (2.7) depends on the choice of $\kappa$ with respect to $v$. To this end, let $Z_{h p} \subset Z$ be a finite dimensional discretization space and determine $\kappa$ such that

$$
\left\langle\kappa, \gamma\left(z_{h p}\right)\right\rangle=\left\langle B v, z_{h p}\right\rangle-\left\langle\ell, z_{h p}\right\rangle
$$

for all $z_{h p} \in Z_{h p}$. Assuming $v \in Z_{h p}$ we observe that $v$ serves as a discretization solution of the variational equation (2.5). Thus, a reliable and efficient a posteriori error estimator $\hat{\eta}$ for the discretization error $\|\hat{v}-v\|_{Z}$ may be available so that (2.6) and (2.7) are fulfilled. For such an error estimator the quantity $\kappa$ must possibly be assumed to be in a certain subspace of $Y^{*}$ (e.g. in an $L^{2}$-space when $Y$ is chosen as an $H^{1}$ - or $H^{1 / 2}$-space). Indeed, in Section 3 we determine $\kappa$ in some discretization spaces in $L^{2}$ by solving local problems or, alternatively, by means of the solution $(v, \kappa)$ of a mixed discretization. 
2.3. How to choose $\mu$ and $\zeta$ ? In order to apply Theorem 2.4 (or Theorem 2.5) for the derivation of efficient and reliable error estimates we have to choose $\mu$ and $\zeta$ such that, first, $\mu \in G^{*}$ and $\zeta \in K_{v}$, second, $\Xi_{\varepsilon}(\mu, \zeta) \leq \Xi_{\varepsilon}(\lambda, \gamma(u-v))($ or $\Xi(\mu, \zeta) \leq \Xi(\lambda, \gamma(u-v)))$ and, third, $\mu$ and $\zeta$ are only determined by the known variables $v$ and $\kappa$, but not by the unknowns $u$ and $\lambda$. Then, $\hat{\eta}^{2}+\Xi_{\varepsilon}(\mu, \zeta)\left(\right.$ or $\left.\hat{\eta}^{2}+\Xi(\mu, \zeta)\right)$ gives an efficient and reliable error estimator in this abstract framework of a posteriori error control.

To this end, we introduce the mapping $l: Y^{*} \rightarrow Y$ as the isometric isomorphism resulting from the Riesz representation theorem, i.e., $(l(\mu), y)_{Y}=\langle\mu, y\rangle$ uniquely holds for all $y \in Y$, where $(\cdot, \cdot)_{Y}$ denotes the inner product of $Y$. Note that $Y^{*}$ is also a Hilbert space with the canonical inner product $(\mu, v)_{Y^{*}}:=(\imath(\mu), \imath(v))_{Y}$, where

$$
\|\mu\|_{Y^{*}}=(\mu, \mu)_{Y^{*}}^{1 / 2}=\|l(\mu)\|_{Y} .
$$

Using the positive part with respect to $G^{*}$ and $G$ (see Appendix A for the general definition of the positive part), we define

$$
\begin{aligned}
\mu^{*} & :=\left(\kappa-\frac{\varepsilon}{2} l^{-1}(\gamma(v)-\psi)\right)_{G^{*}}^{+}, \\
\zeta^{*} & :=\psi-\gamma(v)+\left(-\frac{\varepsilon}{2} l(\kappa)-\psi+\gamma(v)\right)_{G}^{+} .
\end{aligned}
$$

Remark 2.2. It may be convenient to avoid the determination of the positive part with respect to $G^{*}$. It is easy to see that $\imath\left(G^{*}\right)=G_{*}:=\left\{y \in Y \mid \forall w \in G:(y, w)_{Y} \geq 0\right\}$, and hence

$$
\mu^{*}=\imath^{-1}\left(\left(\imath(\kappa)-\frac{\varepsilon}{2}(\gamma(v)-\psi)\right)_{G_{*}}^{+}\right) .
$$

In particular, if the cone $G$ is selfdual, i.e., $G=G_{*}$, the positive part is determined with respect to $G$.

Lemma 2.3. It holds $\mu^{*} \in G^{*}, \zeta^{*} \in K_{v}$ and

$$
\Xi_{\varepsilon}\left(\mu^{*}, \zeta^{*}\right) \leq \Xi_{\varepsilon}(\lambda, \gamma(u-v)) .
$$

If $\mu^{*} \neq \lambda$ or $\zeta^{*} \neq \gamma(u-v)$, then

$$
\Xi_{\varepsilon}\left(\mu^{*}, \zeta^{*}\right)<\Xi_{\varepsilon}(\lambda, \gamma(u-v)) .
$$

Proof. It is clear that $\mu^{*} \in G^{*}$. Moreover, we have

$$
\zeta^{*}+\gamma(v)-\psi=\left(-\frac{\varepsilon}{2} \imath(\kappa)-\psi+\gamma(v)\right)_{G}^{+} \in G,
$$

which shows $\zeta^{*} \in K_{v}$. Defining

$$
M(\mu):=\|\mu-\kappa\|_{Y^{*}}^{2}+\varepsilon\langle\mu, \gamma(v)-\psi\rangle=\|\mu-\kappa\|_{Y^{*}}^{2}+\left(\mu, \varepsilon \imath^{-1}(\gamma(v)-\psi)\right)_{Y^{*}}
$$

and

$$
N(\zeta):=\|\zeta\|_{Y}^{2}+\varepsilon\langle\kappa, \zeta\rangle=\|\zeta\|_{Y}^{2}+(\varepsilon \imath(\kappa), \zeta)_{Y}
$$


we conclude from Lemma A.1 that $M\left(\mu^{*}\right)=\inf _{\mu \in G^{*}} M(\mu)$ and $N\left(\zeta^{*}\right)=\inf _{\zeta \in K_{v}} N(\zeta)$. This together with $\lambda \in G^{*}$ and $\gamma(u-v) \in K_{v}$ immediately gives

$$
\begin{aligned}
\Xi_{\varepsilon}\left(\mu^{*}, \zeta^{*}\right) & =M\left(\mu^{*}\right)+N\left(\zeta^{*}\right) \\
& =\inf _{\mu \in G^{*}} M(\mu)+\inf _{\zeta \in K_{v}} N(\zeta) \\
& =\inf _{\mu \in G^{*}, \zeta \in K_{v}} \Xi_{\varepsilon}(\mu, \zeta) \\
& \leq \Xi_{\varepsilon}(\lambda, \gamma(u-v)),
\end{aligned}
$$

which shows (2.17). Since $\mu \mapsto M(\mu)$ and $\zeta \mapsto N(\zeta)$ are strictly convex, this inequality is strict, when we assume $\mu^{*} \neq \lambda$ or $\zeta^{*} \neq \gamma(u-v)$, which is (2.18).

Theorem 2.6. Let $0<\varepsilon<\min \left\{F\left(E c^{2} \hat{D}\right)^{-1}, \theta^{-1}\right\}$ and $\eta^{2}:=\hat{\eta}^{2}+\Xi_{\varepsilon}\left(\mu^{*}, \zeta^{*}\right)$. Then,

$$
\begin{aligned}
& C \eta^{2} \leq\|u-v\|_{Z}^{2}+\|\lambda-\kappa\|_{Y^{*}}^{2}+\mathrm{osc}^{2}, \\
& \|u-v\|_{Z}^{2}+\|\lambda-\kappa\|_{Y^{*}}^{2} \leq D\left(\eta^{2}+\mathrm{osc}^{2}\right) .
\end{aligned}
$$

Proof. The assertion directly follows from Theorem 2.4 and Lemma 2.3.

Remark 2.3. The error estimator $\eta$ as defined in Theorem 2.6 may have the disadvantage that it depends on the (probably unknown) parameter $\varepsilon$ so that the application of Theorem 2.5 seems to be more appropriate. However, it is unclear how to choose $\mu \in G^{*}$ and $\zeta \in K_{v}$ with $\Xi(\mu, \zeta) \leq \Xi(\lambda, \gamma(u-v))$ in order to achieve a similar result as in Theorem 2.6.

Remark 2.4. The proof of Lemma 2.3 relies on the observation that minimizing the weighted functional $\Xi_{\varepsilon}$ yields some $\mu$ and $\zeta$ with the desired properties as discussed in the beginning of this subsection. This minimizing is done with the help of the general results of Appendix A for minimizing quadratic functionals.

The use of the abstract error estimator $\eta$ in the context of a concrete problem requires that $\mu^{*}$ and $\zeta^{*}$ are computable. Unfortunately, this is not the case for the variational inequalities discussed in Section 3. But, due to the strict inequality in (2.18) it is possible to use some approximations of $\mu^{*}$ and $\zeta^{*}$.

Theorem 2.7. Let $\mu^{*} \neq \lambda$ or $\zeta^{*} \neq \gamma(u-v)$. Furthermore, let $\left\{\mu_{n}^{*}\right\}_{n \in \mathbb{N}} \subset G^{*}$ with $\mu_{n}^{*} \rightarrow \mu^{*}$ and $\left\{\zeta_{n}^{*}\right\}_{n \in \mathbb{N}} \subset K_{v}$ with $\zeta_{n}^{*} \rightarrow \zeta^{*}$ as $n \rightarrow \infty$. Furthermore, let $0<\varepsilon<\min \left\{F\left(E c^{2} \hat{D}\right)^{-1}, \theta^{-1}\right\}$ and $\eta_{n}^{2}:=\hat{\eta}^{2}+\Xi_{\varepsilon}\left(\mu_{n}^{*}, \zeta_{n}^{*}\right)$. Then,

$$
\begin{aligned}
& C \eta_{n}^{2} \leq\|u-v\|_{Z}^{2}+\|\lambda-\kappa\|_{Y^{*}}^{2}+\mathrm{osc}^{2} \\
& \|u-v\|_{Z}^{2}+\|\lambda-\kappa\|_{Y^{*}}^{2} \leq D\left(\eta_{n}^{2}+\mathrm{osc}^{2}\right)
\end{aligned}
$$

for a sufficiently large $n \in \mathbb{N}$.

Proof. Due to Lemma 2.3 and the continuity of $(\mu, \zeta) \mapsto \Xi_{\varepsilon}(\mu, \zeta)$ there exists an $n_{0} \in \mathbb{N}$ such that

$$
\Xi_{\varepsilon}\left(\mu_{n}^{*}, \zeta_{n}^{*}\right)-\Xi_{\varepsilon}\left(\mu^{*}, \zeta^{*}\right)<\Xi_{\varepsilon}(\lambda, \gamma(u-v))-\Xi_{\varepsilon}\left(\mu^{*}, \zeta^{*}\right)
$$

for all $n \geq n_{0}$. This immediately gives $\Xi_{\varepsilon}\left(\mu_{n}^{*}, \zeta_{n}^{*}\right)<\Xi_{\varepsilon}(\lambda, \gamma(u-v))$. Eventually, Theorem 2.4 gives the assertion.

We note some properties of $\mu^{*}$ and $\zeta^{*}$. 
Theorem 2.8. The pair $\left(y^{*}, \mu^{*}\right) \in Y \times G^{*}$ with $y^{*}:=\imath\left(\mu^{*}-\kappa\right)$ uniquely solves the system

$$
\begin{aligned}
& \left(y^{*}, y\right)_{Y}=\left\langle\mu^{*}-\kappa, y\right\rangle, \\
& \left\langle\mu^{*}-\mu, y^{*}+\frac{\varepsilon}{2}(\gamma(v)-\psi)\right\rangle \leq 0
\end{aligned}
$$

for all $(y, \mu) \in Y \times G^{*}$. Moreover,

$$
\left\|\lambda-\mu^{*}\right\|_{Y^{*}} \leq\|\lambda-\kappa\|_{Y^{*}}+\frac{\varepsilon c_{\gamma}}{2}\|u-v\|_{Z}
$$

Proof. Clearly, $\left(y^{*}, \mu^{*}\right)$ solves (2.19a). Furthermore, the positive part in the definition of $\mu^{*}$ gives

$$
\begin{aligned}
0 & \leq\left(\mu^{*}-\kappa+\frac{\varepsilon}{2} l^{-1}(\gamma(v)-\psi), \mu-\mu^{*}\right)_{Y^{*}} \\
& =\left(y^{*}+\frac{\varepsilon}{2}(\gamma(v)-\psi), \imath\left(\mu-\mu^{*}\right)\right)_{Y} \\
& =\left\langle\mu-\mu^{*}, y^{*}+\frac{\varepsilon}{2}(\gamma(v)-\psi)\right\rangle,
\end{aligned}
$$

which means that $\left(y^{*}, \mu^{*}\right)$ also solves (2.19b). Let $\left(\tilde{y}^{*}, \tilde{\mu}^{*}\right) \in Y \times G^{*}$ solve the system (2.19). We immediately get $\tilde{y}^{*}=\imath\left(\tilde{\mu}^{*}-\kappa\right)$. Hence, (2.19b) implies

$$
\begin{aligned}
0 & \geq\left\langle\tilde{\mu}^{*}-\mu, \imath\left(\tilde{\mu}^{*}-\kappa+\frac{\varepsilon}{2} l^{-1}(\gamma(v)-\psi)\right)\right\rangle \\
& =\left(\imath\left(\tilde{\mu}^{*}-\mu\right), \imath\left(\tilde{\mu}^{*}-\kappa+\frac{\varepsilon}{2} l^{-1}(\gamma(v)-\psi)\right)\right)_{Y} \\
& =\left(\tilde{\mu}^{*}-\left(\kappa-\frac{\varepsilon}{2} l^{-1}(\gamma(v)-\psi)\right), \mu-\tilde{\mu}^{*}\right)_{Y^{*}} .
\end{aligned}
$$

Due to the uniqueness of the positive part, we obtain $\tilde{\mu}^{*}=\mu^{*}$ and, thus, $\tilde{y}^{*}=y^{*}$. From (2.4), (2.19b) and Theorem 2.2, we conclude

$$
\begin{aligned}
\left\|\lambda-\mu^{*}\right\|_{Y^{*}}^{2} & =\left(\lambda, \lambda-\mu^{*}\right)_{Y^{*}}-\left(\mu^{*}, \lambda-\mu^{*}\right)_{Y^{*}} \\
& \leq\left(\lambda, \lambda-\mu^{*}\right)_{Y^{*}}-\left(\kappa+\frac{\varepsilon}{2} l^{-1}(\gamma(v)-\psi), \lambda-\mu^{*}\right)_{Y^{*}} \\
& =\left(\lambda-\kappa, \lambda-\mu^{*}\right)_{Y^{*}}+\frac{\varepsilon}{2}\left(\gamma(v)-\psi, \imath\left(\lambda-\mu^{*}\right)\right)_{Y} \\
& =\left(\lambda-\kappa, \lambda-\mu^{*}\right)_{Y^{*}}+\frac{\varepsilon}{2}\left\langle\lambda-\mu^{*}, \gamma(v)-\psi\right\rangle \\
& \leq\left(\lambda-\kappa, \lambda-\mu^{*}\right)_{Y^{*}}+\frac{\varepsilon}{2}\left\langle\lambda-\mu^{*}, \gamma(v-u)\right\rangle \\
& \leq\left(\|\lambda-\kappa\|_{Y^{*}}+\frac{\varepsilon c_{\gamma}}{2}\|u-v\|_{Z}\right)\left\|\lambda-\mu^{*}\right\|_{Y^{*}} .
\end{aligned}
$$

Theorem 2.9. It holds that $\zeta^{*} \in K_{v}$ uniquely solves

$$
\left(\zeta^{*}, y-\zeta^{*}\right)_{Y} \geq\left\langle-\frac{\varepsilon}{2} \kappa, y-\zeta^{*}\right\rangle
$$

for all $y \in K_{v}$. Moreover,

$$
\left\|\zeta^{*}\right\|_{Y} \leq \sqrt{\frac{5}{8}} \varepsilon\|\lambda-\kappa\|_{Y^{*}}+2 c_{\gamma}\|u-v\|_{Z}
$$


Proof. The positive part in the definition of $\zeta^{*}$ implies

$$
\left(\zeta^{*}+\frac{\varepsilon}{2} l(\kappa), w-\zeta^{*}+\psi-\gamma(v)\right)_{Y} \geq 0
$$

for all $w \in G$. Hence, $\zeta^{*}$ solves (2.20). Let $\tilde{\zeta}^{*} \in K_{v}$ also solve (2.20). Then,

$$
\left(\tilde{\zeta}^{*}, \zeta^{*}-\tilde{\zeta}^{*}\right)_{Y} \geq\left\langle-\frac{\varepsilon}{2} \kappa, \zeta^{*}-\tilde{\zeta}^{*}\right\rangle .
$$

Inserting $\tilde{\zeta}^{*}$ in (2.20) and adding to (2.21) give $\left(\tilde{\zeta}^{*}-\zeta^{*}, \zeta^{*}-\tilde{\zeta}^{*}\right)_{Y} \geq 0$, which shows $\tilde{\zeta}^{*}=\zeta^{*}$. Inserting $\gamma(u-v) \in K_{v}$ in (2.20) and exploiting

$$
\left\langle\lambda, \gamma(u-v)-\zeta^{*}\right\rangle=\left\langle\lambda, \psi-\gamma(v)-\zeta^{*}\right\rangle \leq 0
$$

yield

$$
\begin{aligned}
\left\|\zeta^{*}\right\|_{Y}^{2} & \leq\left(\zeta^{*}, \gamma(v-u)\right)_{Y}+\frac{\varepsilon}{2}\left\langle\kappa, \gamma(u-v)-\zeta^{*}\right\rangle \\
& =\left(\zeta^{*}, \gamma(v-u)\right)_{Y}+\frac{\varepsilon}{2}\left\langle\kappa-\lambda, \gamma(u-v)-\zeta^{*}\right\rangle+\frac{\varepsilon}{2}\left\langle\lambda, \gamma(u-v)-\zeta^{*}\right\rangle \\
& \leq \frac{1}{2}\left\|\zeta^{*}\right\|_{Y}^{2}+2 c_{\gamma}^{2}\|u-v\|_{Z}^{2}+\frac{5 \varepsilon^{2}}{16}\|\kappa-\lambda\|_{Y^{*}}^{2},
\end{aligned}
$$

which gives the assertion.

Remark 2.5. There is a strong connection between $\mu^{*}$ and $\lambda$ as well as $\zeta^{*}$ and 0 . In order to see this, let $\left\{\kappa_{n}\right\}_{n \in \mathbb{N}} \subset Y^{*}$ with $\kappa_{n} \rightarrow \lambda$ and $\left\{v_{n}\right\}_{n \in \mathbb{N}} \subset Z$ with $v_{n} \rightarrow u$ as $n \rightarrow \infty$ (for instance, some discretization solutions $v_{n}$ of (2.1) where $\kappa_{n}$ is computed by (2.14) or discretization solutions $\left(v_{n}, \kappa_{n}\right)$ of a mixed discretization so that (2.14) is fulfilled, see Remark (2.1)). Theorem 2.8 and Theorem 2.9 show that $\mu_{n}^{*}$ and $\zeta_{n}^{*}$ as defined in (2.15) and (2.16) with $\kappa_{n}$ and $v_{n}$ for $\kappa$ and $v$ tend to $\lambda$ and 0 as $n \rightarrow \infty$, respectively.

Remark 2.6. The sequences $\left\{\mu_{n}^{*}\right\}_{n \in \mathbb{N}} \subset G^{*}$ with $\mu_{n}^{*} \rightarrow \mu^{*}$ and $\left\{\zeta_{n}^{*}\right\}_{n \in \mathbb{N}} \subset K_{v}$ with $\zeta_{n}^{*} \rightarrow \zeta^{*}$ as $n \rightarrow \infty$ in Theorem 2.7 may result from some discretization of (2.19) as well as (2.20). We refer to Section 3 for more details.

\section{A POSTERIORI ERROR ESTIMATES FOR THE OBSTACLE PROBLEM AND THE SIMPLIFIED SIGNORINI PROBLEM}

3.1. Notations. Let $\Omega \subset \mathbb{R}^{2}$ be a bounded, polygonal Lipschitz domain and $L^{2}(\Omega), H^{1 / 2}(\partial \Omega)$ and $H^{1}(\Omega)$ denote the usual Sobolev spaces. Furthermore, let $H_{D}^{1}(\Omega):=\left\{z \in H^{1}(\Omega)|\gamma(z)|_{D}=\right.$ $0\}$ with a closed subset $\Gamma_{D} \subset \partial \Omega$ with positive measure and the trace operator $\gamma: H^{1}(\Omega) \rightarrow$ $H^{1 / 2}(\partial \Omega)$. We denote its dual space by $H_{D}^{-1}(\Omega):=\left(H_{D}^{1}(\Omega)\right)^{*}$. In the case that $\Gamma_{D} \neq \partial \Omega$ let $\Gamma_{C} \subset \Gamma_{N}:=\partial \Omega \backslash \Gamma_{D}$ be a (relatively) open subset with $\overline{\Gamma_{C}} \subsetneq \overline{\Gamma_{N}}$. Note that $\gamma_{C}: H^{1}(\Omega) \rightarrow$ $H^{1 / 2}\left(\Gamma_{C}\right)$ with $\gamma_{C}(v):=\gamma(v)_{\mid \Gamma_{C}}$ is surjective, see [4, Ch.5].

Let $B: H_{D}^{1}(\Omega) \rightarrow H_{D}^{-1}(\Omega)$ and $\ell \in\left(L^{2}(\Omega)\right)^{*}$ defined as

$$
(B u)(z):=(\nabla u, \nabla z)_{L^{2}(\Omega)}, \quad \ell(z):=(f, z)_{L^{2}(\Omega)}
$$

for $u, z \in H_{D}^{1}(\Omega)$ and $f \in L^{2}(\Omega)$, where $(\cdot, \cdot)_{L^{2}(\Omega)}$ denotes the $L^{2}$-scalar product for scalar or vector-valued $L^{2}$-functions. Due to Cauchy's and Poincare's inequalities we have

$$
\|B z\|_{H_{D}^{-1}(\Omega)} \leq\|z\|_{H^{1}(\Omega)}, \quad\langle B z, z\rangle \geq \beta\|z\|_{H^{1}(\Omega)}
$$


for some constant $\beta>0$ and all $z \in H_{D}^{1}(\Omega)$.

Let $\mathscr{T}_{h}$ be a locally quasi-uniform and $\alpha$-shape regular decomposition of $\Omega$ into triangles and/or parallelograms with global mesh size $h:=\max _{T \in \mathscr{T}_{h}} h_{T}$ with $h_{T}:=\operatorname{diam}(T)$. Furthermore, let $p$ be a polynomial degree distribution on $\mathscr{T}_{h}$ with comparable polynomial degrees of neighboring elements. We refer to [33, Section 1.2] for a definition of locally uniform and $\alpha$-shape regular decompositions and comparable polynomial degrees. Using the polynomial spaces

$$
\mathbb{P}_{p}(T):= \begin{cases}\operatorname{span}\left\{x^{i} y^{j} \mid 0 \leq i+j \leq p\right\}, & T \text { is a triangle } \\ \operatorname{span}\left\{x^{i} y^{j} \mid 0 \leq i, j \leq p\right\}, & T \text { is a parallelogram }\end{cases}
$$

we define the finite element discretization spaces

$$
Z_{h p}:=W_{h p} \cap H_{D}^{1}(\Omega), \quad W_{h p}:=\left\{w_{h p} \in L^{2}(\Omega)\left|\forall T \in \mathscr{T}_{h}: w_{h p}\right|_{T} \circ F_{T} \in \mathbb{P}_{p_{T}}(T)\right\},
$$

where $F_{T}: \widehat{T} \rightarrow T$ is an affine map for $T \in \mathscr{T}_{h}$ with $\widehat{T}:=\{(x, y) \mid 0<x<1,0<y<\sqrt{3}(1 / 2-$ $|1 / 2-x|)\}$ if $T$ is a triangle and $\widehat{T}:=(0,1)^{2}$ if $T$ is a parallelogram. We assume that $\Gamma_{D}$ and $\Gamma_{C}$ are compatible with $\mathscr{T}_{h}$, i.e. $\Gamma_{D}$ and $\Gamma_{C}$ are assumed to be unions of edges of $\mathscr{T}_{h}$. In the following, $h_{e}$ denotes the length of the edge $e \in \mathscr{E}_{h}$ and $p_{e}$ is the polynomial degree on $e$ (e.g. obtained by the minimum rule), where $\mathscr{E}_{h}$ is the set of edges in the interior of $\Omega$ induced by the mesh $\mathscr{T}_{h}$. Moreover, we set $\mathscr{E}_{h} C:=\left\{e \in \mathscr{E}_{h} \mid e \subset \Gamma_{C}\right\}$.

Note that we identify $L^{2}(\Omega)$ and $L^{2}\left(\Gamma_{C}\right)$ with their dual spaces such that $H_{D}^{1}(\Omega) \subset L^{2}(\Omega) \subset$ $H_{D}^{-1}(\Omega)$ and $H^{1 / 2}\left(\Gamma_{C}\right) \subset L^{2}\left(\Gamma_{C}\right) \subset H^{-1 / 2}\left(\Gamma_{C}\right)$, i.e. a function $g \in L^{2}(\Omega)$ or $g \in L^{2}\left(\Gamma_{C}\right)$ is also in $\left(L^{2}(\Omega)\right)^{*}$ or in $\left(L^{2}\left(\Gamma_{C}\right)\right)^{*}$, respectively, if and only if $L^{2}(\Omega) \ni w \mapsto(g, w)_{L^{2}(\Omega)}$ or $L^{2}\left(\Gamma_{C}\right) \ni$ $w \mapsto(g, w)_{L^{2}\left(\Gamma_{C}\right)}$ is in the corresponding dual space.

3.2. A posteriori error estimates for the obstacle problem. The variational formulation of the obstacle problem (see, e.g., [2]) is given by (2.1) with $Z:=H_{D}^{1}(\Omega), B$ and $\ell$ as in (3.1), $K:=\left\{v \in H_{D}^{1}(\Omega) \mid v-\psi \geq 0\right.$ a.e. in $\left.\Omega\right\}$ and $\psi \in H_{D}^{1}(\Omega)$. Note that $K$ is described by linear constraints with $Y:=H_{D}^{1}(\Omega)$,

$$
\begin{aligned}
G & :=\left\{v \in H_{D}^{1}(\Omega) \mid v \geq 0 \text { a.e. in } \Omega\right\}, \\
G^{*} & :=\left\{\mu \in H_{D}^{-1}(\Omega) \mid \forall w \in H_{D}^{1}(\Omega):\langle\mu, w\rangle \geq 0\right\}
\end{aligned}
$$

and $\gamma(v):=v$. From Theorem 2.1, we conclude the unique existence of $u \in H_{D}^{1}(\Omega)$ of the obstacle problem.

We derive a posteriori error estimates for an arbitrary $v \in Z_{h p}$. For this purpose, we choose some $\kappa \in H_{D}^{-1}(\Omega)$ such that

$$
\left\langle\kappa, z_{h p}\right\rangle=\left(\nabla v, \nabla z_{h p}\right)_{L^{2}(\Omega)}-\left(f, z_{h p}\right)_{L^{2}(\Omega)}
$$

for all $z_{h p} \in Z_{h p}$, see Remark 2.1. If $\kappa$ is further assumed to be in $W_{h p}$, this can, for instance, be done by solving (3.4) locally, i.e.,

$$
\left(\kappa_{\mid T}, w\right)_{L^{2}(T)}=(\nabla v, \nabla w)_{L^{2}(T)}-(f, w)_{L^{2}(T)}
$$

for all $T \in \mathscr{T}_{h}$ and all $w \in \mathbb{P}_{p_{T}}$. Alternatively as mentioned in Remark $2.1,(v, \kappa)$ may be the solution of a mixed discretization. For instance, $(v, \kappa)$ is assumed to be in $Z_{h p} \times \Lambda_{H q}$ and to 
fulfill

$$
\begin{aligned}
& \left(\nabla v, \nabla z_{h p}\right)_{L^{2}(\Omega)}=\left(f+\kappa, z_{h p}\right)_{L^{2}(\Omega)} \\
& \left(\mu_{H q}-\kappa, v-\psi\right)_{L^{2}(\Omega)} \geq 0
\end{aligned}
$$

for all $\left(z_{h p}, \mu_{H q}\right) \in Z_{h p} \times \Lambda_{H q}$ with a closed and convex cone $\Lambda_{H q} \subset W_{H q}$ with $0 \in \Lambda_{H q}$ and a discretization space $W_{H q}$ as in (3.2), see [35] for more details of such mixed discretizations. In both cases we assume $v \in Z_{h p}$ and $\kappa \in L^{2}(\Omega)$ and, thus, we can apply an error estimator for the auxiliary problem: find $\hat{v} \in H_{D}^{1}(\Omega)$ such that

$$
(\nabla \hat{v}, \nabla z)=(f+\kappa, z)_{L^{2}(\Omega)}
$$

for all $z \in H_{D}^{1}(\Omega)$, which corresponds to (2.5) and for which $v$ is a discretization solution with respect to $Z_{h p}$, see Remark 2.1. Here, we apply the residual-based error estimator

$$
\begin{aligned}
& \hat{\eta}^{2}:=\sum_{T \in \mathscr{T}_{h}} \hat{\eta}_{T}^{2}, \\
& \hat{\eta}_{T}^{2}:=\frac{h_{T}^{2}}{p_{T}^{2}}\left\|\Pi_{T, p_{T}}(f+\kappa)+\Delta v\right\|_{L^{2}(T)}^{2}+\sum_{e \in \mathscr{E}_{T}} \frac{h_{e}}{2 p_{e}}\left\|\llbracket \partial_{n} v\right\|\left\|_{L^{2}(e)}^{2}+\sum_{e \in \mathscr{E}_{T}} \frac{h_{e}}{2 p_{e}}\right\| \partial_{n} v \|_{L^{2}(e)}^{2},
\end{aligned}
$$

where $\Pi_{T, p_{T}}$ is the $L^{2}(T)$-projection onto the space of polynomials of degree not greater than $p_{T}-1$ and $\mathscr{E}_{T}$ and $\mathscr{E}_{T}^{N}$ denote the set of edges of $T$ in the interior of $\Omega$ or on $\Gamma_{N}$, respectively. From [33, Thm. 3.6], we ascertain that this estimator is reliable up to some oscillations, i.e., there exists a constant $\hat{D}>0$ such that

$$
\|\hat{v}-v\|_{H^{1}(\Omega)}^{2} \leq \hat{D}\left(\hat{\eta}^{2}+\operatorname{osc}^{2}\right)
$$

where the oscillation term is defined as

$$
\operatorname{osc}_{T}^{2}:=\frac{h_{T}^{2}}{p_{T}^{2}}\left\|f+\kappa-\Pi_{T, p_{T}}(f+\kappa)\right\|_{L^{2}(T)}^{2}, \quad \operatorname{osc}^{2}:=\operatorname{osc}^{2}\left(\mathscr{T}_{h}\right):=\sum_{T \in \mathscr{T}_{h}} \operatorname{osc}_{T}^{2} .
$$

Moreover, the estimator is locally efficient (up to oscillations and some $p$-depending efficiency constants), i.e., there exists a constant $\rho(s)$ such that

$$
\hat{\eta}_{T}^{2} \leq \rho(s) p_{T}^{1+2 s}\left(p_{T}\|\hat{v}-v\|_{H^{1}\left(\bigcup \omega_{T}\right)}^{2}+p_{T}^{2 s} \operatorname{osc}^{2}\left(\omega_{T}\right)\right)
$$

for $s>0$ and $T \in \mathscr{T}_{h}$ and $\omega_{T}:=\left\{\hat{T} \in \mathscr{T}_{h} \mid \hat{T}\right.$ and $T$ share at least one edge $\}$. Hence, we have

$$
\begin{aligned}
\sum_{T \in \mathscr{T}_{h}} \hat{\eta}_{T}^{2} & \leq \rho(s) \sum_{T \in \mathscr{T}_{h}} p_{T}^{1+2 s}\left(p_{T}\|\hat{v}-v\|_{H^{1}\left(\cup \omega_{T}\right)}^{2}+p_{T}^{2 s} \operatorname{osc}^{2}\left(\omega_{T}\right)\right) \\
& \leq \rho(s) \max _{T \in \mathscr{T}_{h}}\left(p_{T}^{1+2 s+\max \{1,2 s\}}\right) \sum_{T \in \mathscr{T}_{h}}\left(\|\hat{v}-v\|_{H^{1}\left(\cup \omega_{T}\right)}^{2}+\operatorname{osc}^{2}\left(\omega_{T}\right)\right) \\
& \leq \rho(s) k \max _{T \in \mathscr{T}_{h}}\left(p_{T}^{1+2 s+\max \{1,2 s\}}\right)\left(\|\hat{v}-v\|_{H^{1}(\Omega)}^{2}+\mathrm{osc}^{2}\right)
\end{aligned}
$$

where we exploit that $\#\left\{\omega_{\tilde{T}} \mid T \in \omega_{\tilde{T}}, \tilde{T} \in \mathscr{T}_{h}\right\}$ is bounded for $T \in \mathscr{T}_{h}$ by some $k \in \mathbb{N}$ independent of $h$. Hence,

$$
\hat{C} \hat{\eta}^{2} \leq\|\hat{v}-v\|_{H^{1}(\Omega)}^{2}+\mathrm{osc}^{2}
$$

with

$$
\hat{C}:=\left(\rho(s) p_{T}^{1+2 s+\max \{1,2 s\}} k\right)^{-1}
$$

and, thus, $\hat{\eta}$ fulfills the conditions (2.6) and (2.7). 
We note that the positive part with respect to $\tilde{G}:=\left\{z \in L^{2}(\Omega) \mid z \geq 0\right.$ a.e. in $\left.\Omega\right\}$ is given by the cut-off function

$$
w^{+}:=w_{\tilde{G}}^{+}=\max \{w, 0\}
$$

for $w \in L^{2}(\Omega)$ and provides a continuous mapping $w \mapsto w^{+}$in $L^{2}(\Omega)$ (see (A.2)), but also in $H^{1}(\Omega)$, see [2, Ch.I, Cor.2.1]. Thus, we observe that $\kappa^{+} \in G^{*}$ due to $\left(\kappa^{+}, v\right) \geq 0$ for all $v \in G$ and $(v-\psi)^{+} \in K_{v}:=\left\{\zeta \in H_{D}^{1}(\Omega) \mid \zeta+v-\psi \in G\right\}$ as $(v-\psi)^{+} \in H^{1}(\Omega)$. Thus, Theorem 2.4 and Theorem 2.5 give the reliable error estimates

$$
\|u-v\|_{H^{1}(\Omega)}^{2}+\|\lambda-\kappa\|_{H_{D}^{-1}(\Omega)}^{2} \leq D\left(\hat{\eta}^{2}+\Xi_{\varepsilon}\left(\kappa^{+},(v-\psi)^{+}\right)+\mathrm{osc}^{2}\right)
$$

for some $0<\varepsilon<\min \left\{F\left(E c^{2} \hat{D}\right)^{-1}, \theta^{-1}\right\}$ with

$$
\Xi_{\varepsilon}(\mu, \zeta):=\|\mu-\kappa\|_{H_{D}^{-1}(\Omega)}^{2}+\|\zeta\|_{H^{1}(\Omega)}^{2}+\varepsilon(\mu, v-\psi)_{L^{2}(\Omega)}+\varepsilon(\kappa, \zeta)_{L^{2}(\Omega)}
$$

and

$$
\|u-v\|_{H^{1}(\Omega)}^{2}+\|\lambda-\kappa\|_{H_{D}^{-1}(\Omega)}^{2} \leq \tilde{D}\left(\hat{\eta}^{2}+\Xi\left(\kappa^{+},(v-\psi)^{+}\right)+\mathrm{osc}^{2}\right)
$$

with

$$
\Xi(\mu, \zeta):=\|\mu-\kappa\|_{H_{D}^{-1}(\Omega)}^{2}+\|\zeta\|_{H^{1}(\Omega)}^{2}+\left|(\mu, v-\psi)_{L^{2}(\Omega)}+(\kappa, \zeta)_{L^{2}(\Omega)}\right| .
$$

Unfortunately, the choice of the cut-off functions $\kappa^{+}$and $(v-\psi)^{+}$(for $\mu$ and $\zeta$, see Section 2.3) may not lead to efficient error estimates as the conditions (2.9) and (2.12) may not be ensured for $\lambda \in H_{D}^{-1}(\Omega)$ uniquely fulfilling

$$
\langle\lambda, z\rangle=(\nabla u, \nabla z)+(f, z)_{L^{2}(\Omega)}
$$

for all $z \in H_{D}^{1}(\Omega)$. The use of $\mu^{*}$ and $\zeta^{*}$ as defined in (2.15) and (2.16) together Theorem 2.6 gives the reliable and efficient estimates

$$
\begin{aligned}
& C \eta^{2} \leq\|u-v\|_{H^{1}(\Omega)}^{2}+\|\lambda-\kappa\|_{H_{D}^{-1}(\Omega)}^{2}+\mathrm{osc}^{2} \\
& \|u-v\|_{H^{1}(\Omega)}^{2}+\|\lambda-\kappa\|_{H_{D}^{-1}(\Omega)}^{2} \leq D\left(\eta^{2}+\mathrm{osc}^{2}\right)
\end{aligned}
$$

with $\eta$ defined in this theorem, but $\mu^{*}$ and $\zeta^{*}$ are not computable (in contrast to $\kappa^{+}$and ( $v-$ $\psi)^{+}$). Theorem 2.8 and Theorem 2.9 imply that they (uniquely) fulfill

$$
\begin{aligned}
& a\left(y^{*}, y\right)=\left\langle\mu^{*}, y\right\rangle-(\kappa, y)_{L^{2}(\Omega)} \\
& \left\langle\mu^{*}-\mu, y^{*}+\frac{\varepsilon}{2}(v-\psi)\right\rangle \leq 0
\end{aligned}
$$

for all $(y, \mu) \in H_{D}^{1}(\Omega) \times G^{*}$ and some unique $y^{*} \in H_{D}^{1}(\Omega)$ and

$$
a\left(\zeta^{*}, y-\zeta^{*}\right) \geq\left(-\frac{\varepsilon}{2} \kappa, y-\zeta^{*}\right)_{L^{2}(\Omega)}
$$

for all $y \in K_{v}$. Here, the bilinear form $a$ is defined as

$$
a(\zeta, y):=(\nabla \zeta, \nabla y)_{L^{2}(\Omega)}+(\zeta, y)_{L^{2}(\Omega)}
$$

for $\zeta, y \in H_{D}^{1}(\Omega)$. In order to get computable efficient and reliable error estimates we apply Theorem 2.7 and consider discretizations of (3.12) and (3.13), i.e. find $\left(y_{\bar{h} \bar{p}}^{*}, \mu_{\bar{H} \bar{q}}^{*}\right) \in Z_{\bar{h} \bar{p}} \times \Lambda_{\bar{H} \bar{q}}$ 
such that

$$
\begin{aligned}
& a\left(y_{\bar{h} \bar{p}}^{*}, y_{\bar{h} \bar{p}}\right)_{L^{2}(\Omega)}=\left(\mu_{\bar{H} \bar{q}}^{*}-\kappa, y_{\bar{h} \bar{p}}\right)_{L^{2}(\Omega)} \\
& \left(\mu_{\bar{H} \bar{q}}^{*}-\mu_{\bar{H} \bar{q}}, y_{\bar{h} \bar{p}}^{*}+\frac{\varepsilon}{2}(v-\psi)\right)_{L^{2}(\Omega)} \leq 0
\end{aligned}
$$

for all $\left(y_{\bar{h} \bar{p}}, \mu_{\bar{H} \bar{q}}\right) \in Z_{\bar{h} \bar{p}} \times \Lambda_{\bar{H} \bar{q}}$ where $Z_{\bar{h} \bar{p}}$ and $W_{\bar{H} \bar{q}}$ are some discretization spaces as defined in (3.2) and $\Lambda_{\bar{H} \bar{q}} \subset W_{\bar{H} \bar{q}}$ is a closed and convex cone with $0 \in \Lambda_{\bar{H} \bar{q}}$. Furthermore, find $\zeta_{\bar{h} \bar{q}}^{*} \in K_{v, \bar{h} \bar{p}}$ such that

$$
a\left(\zeta_{\bar{h} \bar{p}}^{*}, y_{\bar{h} \bar{p}}-\zeta_{\bar{h} \bar{p}}^{*}\right) \geq\left(-\frac{\varepsilon}{2} \kappa, y_{\bar{h} \bar{p}}-\zeta_{\bar{h} \bar{p}}^{*}\right)_{L^{2}(\Omega)}
$$

for all $y_{\bar{h} \bar{p}} \in K_{v, \bar{h} \bar{p}}$, where $K_{v, \bar{h} \bar{p}}$ is a closed and convex subset of $Z_{h p}$. We refer to [3, 35, 36] on convergence results and on the existence of discretization solutions and the specific construction of $\Lambda_{\bar{H} \bar{q}}$ and $K_{v, \bar{h} \bar{p}}$.

Theorem 3.1. Let $\mu^{*} \neq \lambda$ or $\zeta^{*} \neq u-v$. Furthermore, let $\left\|\mu^{*}-\mu_{\bar{H} \bar{q}}^{*}\right\|_{L^{2}(\Omega)} \rightarrow 0$ as $\bar{H} / \bar{q} \rightarrow 0$ and $\left\|\zeta_{\bar{h} \bar{p}}^{*}-\zeta^{*}\right\|_{H^{1}(\Omega)} \rightarrow 0$ as $\bar{h} / \bar{p} \rightarrow 0$ and define

$$
\bar{\eta}^{2}:=\hat{\eta}^{2}+\Xi_{\varepsilon}\left(\left(\mu_{\bar{H} \bar{q}}^{*}\right)^{+}, \max \left\{\zeta_{\bar{h} \bar{p}}^{*}, \psi-v\right\}\right) .
$$

Then,

$$
\begin{aligned}
& C \bar{\eta}^{2} \leq\|u-v\|_{H^{1}(\Omega)}^{2}+\|\lambda-\kappa\|_{H_{D}^{-1}(\Omega)}^{2}+\mathrm{osc}^{2} \\
& \|u-v\|_{H^{1}(\Omega)}^{2}+\|\lambda-\kappa\|_{H_{D}^{-1}(\Omega)}^{2} \leq D\left(\bar{\eta}^{2}+\mathrm{osc}^{2}\right),
\end{aligned}
$$

for sufficiently small $\bar{H} / \bar{q}$ and $\bar{h} / \bar{p}$.

Proof. From (A.2) and $\mu^{*} \in \tilde{G}$, we conclude

$$
\left\|\mu^{*}-\left(\mu_{\bar{H} \bar{q}}^{*}\right)^{+}\right\|_{H_{D}^{-1}(\Omega)} \leq\left\|\left(\mu^{*}\right)^{+}-\left(\mu_{\bar{H} \bar{q}}^{*}\right)^{+}\right\|_{L^{2}(\Omega)} \leq\left\|\mu^{*}-\mu_{\bar{H} \bar{q}}^{*}\right\|_{L^{2}(\Omega)} \rightarrow 0
$$

as $\bar{H} / \bar{q} \rightarrow 0$. Obviously, we have

$$
\left\|\left(\zeta^{*}-\psi+v\right)-\left(\zeta_{\bar{h} \bar{p}}^{*}+v-\psi\right)\right\|_{H^{1}(\Omega)}=\left\|\zeta^{*}-\zeta_{\bar{h} \bar{p}}^{*}\right\|_{H^{1}(\Omega)} \rightarrow 0
$$

as $\bar{h} / \bar{p} \rightarrow 0$. Thus, from the continuity of the positive part in $H^{1}(\Omega)$ and $\zeta^{*}-\psi+v \in \tilde{G}$, we conclude that

$$
\begin{aligned}
\left\|\zeta^{*}-\max \left\{\zeta_{\bar{h} \bar{p}}^{*}, \psi-v\right\}\right\|_{H^{1}(\Omega)} & =\left\|\zeta^{*}-\left(\left(\zeta_{\bar{h} \bar{p}}^{*}+v-\psi\right)^{+}-v+\psi\right)\right\|_{H^{1}(\Omega)} \\
& =\left\|\left(\zeta^{*}+v-\psi\right)-\left(\zeta_{\bar{h} \bar{p}}^{*}+v-\psi\right)^{+}\right\|_{H^{1}(\Omega)} \\
& =\left\|\left(\zeta^{*}-\psi+v\right)^{+}-\left(\zeta_{\bar{h} \bar{p}}^{*}+v-\psi\right)^{+}\right\|_{H^{1}(\Omega)} \rightarrow 0
\end{aligned}
$$

as $\bar{h} / \bar{p} \rightarrow 0$. Since $\left(\mu_{\bar{H} \bar{q}}^{*}\right)^{+} \in G^{*}$ and $\max \left\{\zeta_{\bar{h}}^{*}, \psi-v\right\} \in K_{v}$, Theorem 2.7 yields the assertion.

Remark 3.1. We use the positive part $(\cdot)^{+}$with respect $\tilde{G}$ in $L^{2}(\Omega)$ as it is computable. In principle, using the positive parts $(\cdot)_{G}^{+}$and $(\cdot)_{G^{*}}^{+}$would lead to similar results, but these positive parts are not directly evaluable. 
Remark 3.2. Instead of the $\|\cdot\|_{H^{1}(\Omega)}$-norm we may equip $H^{1}(\Omega)$ with a norm, which is induced by the operator $B$ or a part of this operator. For instance, in the case of the obstacle problem we may equip $H^{1}(\Omega)$ with the norm $|\cdot|_{H^{1}(\Omega)}:=\|\nabla \cdot\|_{L^{2}(\Omega)}$ (which is indeed a norm as $\Gamma_{D}$ has a positive measure). In this case the bilinear form $a(\cdot, \cdot)$ in $(3.14)$ reduces to $a(\zeta, y):=$ $(\nabla \zeta, \nabla y)_{L^{2}(\Omega)}$ which possibly has the advantange that the stiffness matrix resulting from the computation of a discretization solution can be reused to compute $\mu_{\bar{H} \bar{q}}^{*}$ and $\zeta_{\bar{h} \bar{p}}^{*}$. Using the same arguments as in Theorem 3.1 we obtain the same error estimates as in this theorem, but for the $|\cdot|_{H^{1}(\Omega)}$-norm and the corresponding dual norm. Due to its equivalence to the $\|\cdot\|_{H^{1}(\Omega)^{-}}$ norm we can transfer these estimates again to the $\|\cdot\|_{H^{1}(\Omega)}$-norm by taking into account the equivalence constants.

Remark 3.3. It is easy to see that $\left(y_{\bar{h} \bar{p}}^{*}, \mu_{\bar{H} \bar{q}}^{*}\right):=(0, \kappa)$ fulfills $(3.15)$ provided that $(v, \kappa)$ is the solution of the mixed discretization (3.5) and that the corresponding discretization spaces coincide, i.e. $Z_{h p}=Z_{\bar{h} \bar{p}}$ and $\Lambda_{H q}=\Lambda_{\bar{H} \bar{q}}$. Moreover, it is also easy to see that $\zeta_{\bar{h} \bar{p}}:=0$ fulfills (3.16) if $K_{v, \bar{h} \bar{p}}=v+K_{h p}$ and if $v \in K_{h p}$ is the solution of the primal discretization

$$
\left(\nabla v, \nabla\left(v-z_{h p}\right)\right)_{L^{2}(\Omega)} \geq\left(f, v-z_{h p}\right)_{L^{2}(\Omega)}
$$

for all $z_{h p} \in K_{h p}$, where $K_{h p}$ is a closed and convex subset of $Z_{h p}$. These observations can be exploited for a practical determination of $\mu_{\bar{H} \bar{q}}^{*}$ and $\zeta_{\bar{h} \bar{p}}^{*}$ : If $v$ results from a mixed discretization of the form (3.5) we may take $\mu_{\bar{H} \bar{q}}^{*}:=\kappa$. If $v$ results from a primal discretization of the form (3.17) we may take $\zeta_{\bar{H} \bar{q}}^{*}:=0$. Here, we have to assume that $H / q$ or $h / p$ is small enough so that Theorem 3.1 yields an efficient error estimator. In any case, these choices seem to be reasonable at least as initial guesses for an iterative solving of (3.15) and (3.16), see also Remark 2.5. In the numerical experiments of Section 4 only few (2 to 5) iterations of an active set solution scheme were neccessary for the computation of $\mu_{\bar{H} \bar{q}}^{*}$ or $\zeta_{\bar{h} \bar{p}}^{*}$. Note that if the mixed discretization (3.5) is equivalent to the primal discretization (3.17) (in the sense that they have the same unique primal solution), we may take both $\mu_{\bar{H} \bar{q}}^{*}:=\kappa$ and $\zeta_{\bar{H} \bar{q}}^{*}:=0$ (which incidently gives the reliable error estimate (3.11)) and may avoid any additional computations. We refer to [24] where a mixed discretization of the obstacle problem with biorthogonal basis functions is discussed. This discretization has the form (3.5) and is, indeed, equivalent to a primal discretization of the form (3.17).

3.3. A posteriori error estimates for the simplified Signorini problem. The variational formulation of the simplified Signorini problem (see, e.g., [2]) is given by (2.1) with $Z:=H_{D}^{1}(\Omega$ ), $B$ and $\ell$ as in (3.1), $K:=\left\{v \in H_{D}^{1}(\Omega) \mid \gamma_{C}(v)-\psi \geq 0\right.$ a.e. on $\left.\Gamma_{C}\right\}$ and $\psi \in H^{1 / 2}\left(\Gamma_{C}\right)$. Note that $K$ is described by linear constraints with $Y:=H^{1 / 2}\left(\Gamma_{C}\right)$,

$$
\begin{aligned}
G & :=\left\{v \in H^{1 / 2}\left(\Gamma_{C}\right) \mid v \geq 0 \text { a.e. on } \Gamma_{C}\right\}, \\
G^{*} & :=\left\{\mu \in H^{-1 / 2}\left(\Gamma_{C}\right) \mid \forall w \in H^{1 / 2}\left(\Gamma_{C}\right):\langle\mu, w\rangle \geq 0\right\}
\end{aligned}
$$

and $\gamma:=\gamma_{C}$. Again, from Theorem 2.1, we conclude the unique existence of $u \in H_{D}^{1}(\Omega)$ of the simplified Signorini problem.

The application of the abstract framework of Section 2 to the simplied Signorini problem is very similar to Section 3.2. Therefore, we repeat this section with some small modifications for 
the derivation of a posteriori error estimates. Let $v \in Z_{h p}$ and $\kappa \in H^{-1 / 2}\left(\Gamma_{C}\right)$ such that

$$
\left\langle\kappa, \gamma_{C}\left(z_{h p}\right)\right\rangle=\left(\nabla v, z_{h p}\right)_{L^{2}(\Omega)}-\left(f, z_{h p}\right)_{L^{2}(\Omega)}
$$

for all $z_{h p} \in Z_{h p}$, see Remark 2.1. Further assuming $\kappa$ in

$$
W_{C, h p}:=\left\{w_{h p \mid \Gamma_{C}} \mid w_{h p} \in W_{h p}\right\}
$$

we can solve (3.18) in a local sense, i.e.,

$$
\left(\kappa_{\mid e}, \gamma_{C}(w)\right)_{L^{2}(e)}=(\nabla v, \nabla w)_{L^{2}(T)}-(f, w)_{L^{2}(T)}
$$

for all $T \in \mathscr{T}_{h}$, all $e \in \mathscr{E}_{h}^{C}$ with $e \subset \partial T$ and all $w \in \mathbb{P}_{p_{T}}$. Alternatively (see Remark 2.1), (v, $\left.\kappa\right)$ may be the solution of a mixed discretization and may fulfill

$$
\begin{aligned}
& \left(\nabla v, \nabla z_{h p}\right)_{L^{2}(\Omega)}=\left(f, z_{h p}\right)_{L^{2}(\Omega)}+\left(\kappa, \gamma_{C}\left(z_{h p}\right)\right)_{L^{2}\left(\Gamma_{C}\right)} \\
& \left(\mu_{H q}-\kappa, \gamma_{C}(v)-\psi\right)_{L^{2}\left(\Gamma_{C}\right)} \geq 0
\end{aligned}
$$

for all $\left(z_{h p}, \mu_{H q}\right) \in Z_{h p} \times \Lambda_{H q}$ with a closed and convex cone $\Lambda_{H q} \subset W_{C, H q}$ with $0 \in \Lambda_{H q}$ and a discretization space $W_{C, H q}$ as in (3.19). In both cases we have $v \in Z_{h p}$ and $\kappa \in L^{2}\left(\Gamma_{C}\right)$ and can apply an error estimator for the auxiliary problem: find $\hat{v} \in H_{D}^{1}(\Omega)$ such that

$$
(\nabla \hat{v}, \nabla z)=(f, z)_{L^{2}(\Omega)}+\left(\kappa, \gamma_{C}(z)\right)_{L^{2}\left(\Gamma_{C}\right)}
$$

for all $z \in H_{D}^{1}(\Omega)$. Note that (3.20) corresponds to (2.5) and $v$ is a discretization solution with respect to $Z_{h p}$, see Remark 2.1. The residual-based error estimator has the form

$$
\begin{aligned}
\hat{\eta}^{2}:= & \sum_{T \in \mathscr{T}_{h}} \hat{\eta}_{T}^{2}, \\
\hat{\eta}_{T}^{2}:= & \frac{h_{T}^{2}}{p_{T}^{2}}\left\|\Pi_{T, p_{T}}(f)+\Delta v\right\|_{L^{2}(T)}^{2}+\sum_{e \in \mathscr{E}_{T}} \frac{h_{e}}{2 p_{e}}\left\|\llbracket \partial_{n} v \rrbracket\right\|_{L^{2}(e)}^{2}+\sum_{e \in \mathscr{E}_{T}^{N} \backslash \mathscr{E}_{T}} \frac{h_{e}}{p_{e}}\left\|\partial_{n} v\right\|_{L^{2}(e)}^{2} \\
& +\sum_{e \in \mathscr{E}_{T}} \frac{h_{e}}{p_{e}}\left\|\kappa-\partial_{n} v\right\|_{L^{2}(e)}^{2},
\end{aligned}
$$

where $\mathscr{E}_{T}^{C}$ denotes the set of edges of $T$ on $\Gamma_{C}$. This estimator is reliable up to some oscillations (see [33, Thm. 3.6]), i.e., there exists a constant $\hat{D}>0$ such that

$$
\|\hat{v}-v\|_{H^{1}(\Omega)}^{2} \leq \hat{D}\left(\hat{\eta}^{2}+\operatorname{osc}^{2}\right)
$$

with

$$
\operatorname{osc}_{T}^{2}:=\frac{h_{T}^{2}}{p_{T}^{2}}\left\|f-\Pi_{T, p_{T}}(f)\right\|_{L^{2}(T)}^{2}, \quad \operatorname{osc}^{2}:=\operatorname{osc}^{2}\left(\mathscr{T}_{h}\right):=\sum_{T \in \mathscr{T}_{h}} \operatorname{osc}_{T}^{2} .
$$

As in the derivation of (3.8) we conclude from the local efficiency of $\hat{\eta}$

$$
\hat{C} \hat{\eta}^{2} \leq\|\hat{v}-v\|_{H^{1}(\Omega)}^{2}+\operatorname{osc}^{2}
$$

with $\hat{C}$ defined in (3.9). Thus, $\hat{\eta}$ fulfills the conditions (2.6) and (2.7).

We use the positive part with respect to $\tilde{G}_{C}:=\left\{z \in L^{2}\left(\Gamma_{C}\right) \mid z \geq 0\right.$ a.e. on $\left.\Gamma_{C}\right\}$ in $L^{2}\left(\Gamma_{C}\right)$, which is given by the cut-off function

$$
w^{+}:=w_{\tilde{G}_{C}}^{+}=\max \{w, 0\}
$$


for $w \in L^{2}\left(\Gamma_{C}\right)$ and which provides a continuous mapping $w \mapsto w^{ \pm}$in $L^{2}\left(\Gamma_{C}\right)$ (see (A.2)), but also in $H^{1}\left(\Gamma_{C}\right)$, see [2, Ch.I, Cor.2.1]. Observing $\kappa^{+} \in G^{*}$ as $\left(\kappa^{+}, w\right)_{L^{2}\left(\Gamma_{C}\right)} \geq 0$ for all $w \in G$ and $\left(\gamma_{C}(v)-\psi\right)^{+} \in K_{v}:=\left\{\zeta \in H^{1 / 2}\left(\Gamma_{C}\right) \mid \zeta+\gamma_{C}(v)-\psi \in G\right\}$ as $\left(\gamma_{C}(v)-\psi\right)^{+} \in H^{1}\left(\Gamma_{C}\right)$ (provided that $\psi \in H^{1}\left(\Gamma_{C}\right)$ ) and applying Theorem 2.4 and Theorem 2.5 we obtain the reliable error estimates

$$
\|u-v\|_{H^{1}(\Omega)}^{2}+\|\lambda-\kappa\|_{H^{-1 / 2}\left(\Gamma_{C}\right)}^{2} \leq D\left(\hat{\eta}^{2}+\Xi_{\varepsilon}\left(\kappa^{+},(v-\psi)^{+}\right)+\operatorname{osc}^{2}\right)
$$

for some $0<\varepsilon<\min \left\{F\left(E c^{2} \hat{D}\right)^{-1}, \theta^{-1}\right\}$ with

$$
\Xi_{\varepsilon}(\mu, \zeta):=\|\mu-\kappa\|_{H^{-1 / 2}\left(\Gamma_{C}\right)}^{2}+\|\zeta\|_{H^{1 / 2}\left(\Gamma_{C}\right)}^{2}+\varepsilon(\mu, v-\psi)_{L^{2}\left(\Gamma_{C}\right)}+\varepsilon(\kappa, \zeta)_{L^{2}\left(\Gamma_{C}\right)}
$$

and

$$
\|u-v\|_{H^{1}\left(\Gamma_{C}\right)}^{2}+\|\lambda-\kappa\|_{H^{-1 / 2}\left(\Gamma_{C}\right)}^{2} \leq \tilde{D}\left(\hat{\eta}^{2}+\Xi\left(\kappa^{+},(v-\psi)^{+}\right)+\mathrm{osc}^{2}\right)
$$

with

$$
\Xi(\mu, \zeta):=\|\mu-\kappa\|_{H_{D}^{-1}(\Omega)}^{2}+\|\zeta\|_{H^{1 / 2}\left(\Gamma_{C}\right)}^{2}+\left|(\mu, v-\psi)_{L^{2}\left(\Gamma_{C}\right)}+(\kappa, \zeta)_{L^{2}\left(\Gamma_{C}\right)}\right|
$$

Again, we note that the choice $\kappa^{+}$and $(v-\psi)^{+}$(for $\mu$ and $\zeta$, see Section 2.3) may not lead to efficient error estimates as the conditions (2.9) and (2.12) may not be guaranteed for $\lambda \in$ $H^{-1 / 2}\left(\Gamma_{C}\right)$ uniquely fulfilling

$$
\langle\lambda, z\rangle=(\nabla u, \nabla z)+(f, z)_{L^{2}(\Omega)}
$$

for all $z \in H_{D}^{1}(\Omega)$. Again, the use of $\mu^{*}$ and $\zeta^{*}$ as defined in (2.15) and (2.16) together with Theorem 2.6 would give the reliable and efficient estimates

$$
\begin{aligned}
& C \eta^{2} \leq\|u-v\|_{H^{1}(\Omega)}^{2}+\|\lambda-\kappa\|_{H^{-1 / 2}\left(\Gamma_{C}\right)}^{2}+\mathrm{osc}^{2} \\
& \|u-v\|_{H^{1}(\Omega)}^{2}+\|\lambda-\kappa\|_{H^{-1 / 2}\left(\Gamma_{C}\right)}^{2} \leq D\left(\eta^{2}+\mathrm{osc}^{2}\right),
\end{aligned}
$$

where $\eta$ is defined as in Theorem 2.6. We note, however, that $\mu^{*}$ and $\zeta^{*}$ are not computable. Theorem 2.8 and Theorem 2.9 imply that they (uniquely) fulfill

$$
\begin{aligned}
& a\left(y^{*}, y\right)=\left\langle\mu^{*}, \gamma_{C}(y)\right\rangle-\left(\kappa, \gamma_{C}(y)\right)_{L^{2}\left(\Gamma_{C}\right)} \\
& \left\langle\mu^{*}-\mu, \gamma_{C}\left(y^{*}\right)+\frac{\varepsilon}{2}\left(\gamma_{C}(v)-\psi\right)\right\rangle \leq 0
\end{aligned}
$$

for all $(y, \mu) \in H_{D}^{1}(\Omega) \times G^{*}$ and some unique $y^{*} \in H_{D}^{1}(\Omega)$ and

$$
a\left(\zeta^{*}, y-\zeta^{*}\right) \geq\left(-\frac{\varepsilon}{2} \kappa, \gamma_{C}(y)-\zeta^{*}\right)_{L^{2}\left(\Gamma_{C}\right)}
$$

for all $y \in K_{v}$, where the bilinear form $a$ is defined in (3.14). In order to get computable efficient and reliable error estimates we apply Theorem 2.7 and consider discretizations of (3.22) and (3.23), i.e. find $\left(y_{\bar{h} \bar{p}}^{*}, \mu_{\bar{H} \bar{q}}^{*}\right) \in Z_{\bar{h} \bar{p}} \times \Lambda_{\bar{H} \bar{q}}$ such that

$$
\begin{aligned}
& a\left(y_{\bar{h} \bar{p}}^{*}, y_{\bar{h} \bar{p}}\right)_{L^{2}(\Omega)}=\left(\mu_{\bar{H} \bar{q}}^{*}-\kappa, \gamma_{C}\left(y_{\bar{h} \bar{p}}\right)\right)_{L^{2}\left(\Gamma_{C}\right)} \\
& \left(\mu_{\bar{H} \bar{q}}^{*}-\mu_{\bar{H} \bar{q}}, \gamma_{C}\left(y_{\bar{h} \bar{p}}^{*}\right)+\frac{\varepsilon}{2}\left(\gamma_{C}(v)-\psi\right)\right)_{L^{2}\left(\Gamma_{C}\right)} \leq 0
\end{aligned}
$$


for all $\left(y_{\bar{h} \bar{p}}, \mu_{\bar{H} \bar{q}}\right) \in Z_{\bar{h} \bar{p}} \times \Lambda_{\bar{H} \bar{q}}$ where $Z_{\bar{h} \bar{p}}$ and $W_{C, \bar{H} \bar{q}}$ are some discretization spaces as defined in (3.2) and (3.19) and $\Lambda_{\bar{H} \bar{q}} \subset W_{C, \bar{H} \bar{q}}$ is a closed and convex cone with $0 \in \Lambda_{\bar{H} \bar{q}}$. Furthermore, find $\zeta_{\bar{h} \bar{q}}^{*} \in K_{v, \bar{h} \bar{p}}$ such that

$$
a\left(\zeta_{\bar{h} \bar{p}}^{*}, y_{\bar{h} \bar{p}}-\zeta_{\bar{h} \bar{p}}^{*}\right) \geq\left(-\frac{\varepsilon}{2} \kappa, \gamma_{C}\left(y_{\bar{h} \bar{p}}\right)-\zeta_{\bar{h} \bar{p}}^{*}\right)_{L^{2}(\Omega)}
$$

for all $y_{\bar{h} \bar{p}} \in K_{v, \bar{h} \bar{p}}$, where $K_{v, \bar{h} \bar{p}}$ is a closed and convex subset of $Z_{h p}$. We refer to $[3,35,37]$ on convergence results, the existence of discretization solutions and the specific construction of $\Lambda_{\bar{H} \bar{q}}$ and $K_{v, \bar{h} \bar{p}}$. See Remark 3.3 for some comments on how to solve (3.24) and (3.25).

Theorem 3.2. Let $\mu^{*} \neq \lambda$ or $\zeta^{*} \neq \gamma_{C}(u-v)$ and let $z_{\psi} \in C^{0}(\bar{\Omega}) \cap H_{D}^{1}(\Omega)$ with $\gamma_{C}\left(z_{\psi}\right)=\psi$ and $\gamma_{C}\left(\zeta^{*}+v-z_{\psi}\right)^{+}=\gamma_{C}\left(\left(\zeta^{*}+v-z_{\psi}\right)^{+}\right)$. Furthermore, let $\left\|\mu^{*}-\mu_{\bar{H} \bar{q}}^{*}\right\|_{L^{2}\left(\Gamma_{C}\right)} \rightarrow 0$ as $\bar{H} / \bar{q} \rightarrow 0$ and $\left\|\zeta_{\bar{h} \bar{p}}^{*}-\zeta^{*}\right\|_{\left.H^{1} \Omega\right)} \rightarrow 0$ as $\bar{h} / \bar{p} \rightarrow 0$ and define

$$
\bar{\eta}^{2}:=\hat{\eta}^{2}+\Xi_{\mathcal{E}}\left(\left(\mu_{\bar{H} \bar{q}}^{*}\right)^{+}, \max \left\{\gamma_{C}\left(\zeta_{\bar{h} \bar{p}}^{*}\right), \psi-\gamma_{C}(v)\right\}\right) .
$$

Then,

$$
\begin{aligned}
& C \bar{\eta}^{2} \leq\|u-v\|_{H^{1}(\Omega)}^{2}+\|\lambda-\kappa\|_{H_{D}^{-1}(\Omega)}^{2}+\mathrm{osc}^{2} \\
& \|u-v\|_{H^{1}(\Omega)}^{2}+\|\lambda-\kappa\|_{H_{D}^{-1}(\Omega)}^{2} \leq D\left(\bar{\eta}^{2}+\mathrm{osc}^{2}\right)
\end{aligned}
$$

for sufficiently small $\bar{H} / \bar{q}$ and $\bar{h} / \bar{p}$.

Proof. From (A.2) and $\mu^{*} \in \tilde{G}_{C}$, we conclude

$$
\left\|\mu^{*}-\left(\mu_{\bar{H} \bar{q}}^{*}\right)^{+}\right\|_{H^{-1 / 2}\left(\Gamma_{C}\right)} \leq\left\|\left(\mu^{*}\right)^{+}-\left(\mu_{\bar{H} \bar{q}}^{*}\right)^{+}\right\|_{L^{2}\left(\Gamma_{C}\right)} \leq\left\|\mu^{*}-\mu_{\bar{H} \bar{q}}^{*}\right\|_{L^{2}\left(\Gamma_{C}\right)} \rightarrow 0
$$

as $\bar{H} / \bar{q} \rightarrow 0$. Obviously, we have

$$
\left\|\left(\zeta^{*}+v-z_{\psi}\right)-\left(\zeta_{\bar{h} \bar{p}}^{*}+v-z_{\psi}\right)\right\|_{H^{1}(\Omega)}=\left\|\zeta^{*}-\zeta_{\bar{h} \bar{p}}^{*}\right\|_{H^{1}(\Omega)} \rightarrow 0
$$

as $\bar{h} / \bar{p} \rightarrow 0$. Thus, from the continuity of the positive part in $H^{1}(\Omega), \zeta_{\bar{h} \bar{p}}^{*}+v-z_{\psi} \in C^{0}(\bar{\Omega}) \cap$ $H_{D}^{1}(\Omega)$ and $\gamma_{C}\left(\zeta^{*}\right)+\gamma_{C}(v)-\psi \in \tilde{G}_{C}$, we conclude that

$$
\begin{aligned}
& \left\|\gamma_{C}\left(\zeta^{*}\right)-\max \left\{\gamma_{C}\left(\zeta_{\bar{h} \bar{p}}^{*}\right), \psi-\gamma_{C}(v)\right\}\right\|_{H^{1 / 2}\left(\Gamma_{C}\right)} \\
& =\left\|\gamma_{C}\left(\zeta^{*}\right)-\left(\left(\gamma_{C}\left(\zeta_{\bar{h} \bar{p}}^{*}\right)+\gamma_{C}(v)-\psi\right)^{+}-\gamma_{C}(v)+\psi\right)\right\|_{H^{1 / 2}\left(\Gamma_{C}\right)} \\
& =\left\|\left(\gamma_{C}\left(\zeta^{*}\right)+\gamma_{C}(v)-\psi\right)-\left(\gamma_{C}\left(\zeta_{\bar{h} \bar{p}}^{*}\right)+\gamma_{C}(v)-\psi\right)^{+}\right\|_{H^{1 / 2}\left(\Gamma_{C}\right)} \\
& =\left\|\left(\gamma_{C}\left(\zeta^{*}\right)+\gamma_{C}(v)-\psi\right)^{+}-\left(\gamma_{C}\left(\zeta_{\bar{h} \bar{p}}^{*}\right)+\gamma_{C}(v)-\psi\right)^{+}\right\|_{H^{1 / 2}\left(\Gamma_{C}\right)} \\
& =\|\left(\gamma_{C}\left(\left(\zeta^{*}+v-z_{\psi}\right)^{+}\right)-\gamma_{C}\left(\left(\zeta_{\bar{h} \bar{p}}^{*}+v-z_{\psi}\right)^{+}\right) \|_{H^{1 / 2}\left(\Gamma_{C}\right)}\right. \\
& \left.\leq c_{\gamma_{C}} \|\left(\zeta^{*}+v-z_{\psi}\right)^{+}-\left(\zeta_{\bar{h} \bar{p}}^{*}+v-z_{\psi}\right)^{+}\right) \|_{H^{1}(\Omega)} \rightarrow 0
\end{aligned}
$$

as $\bar{h} / \bar{p} \rightarrow 0$, where $c_{\gamma_{C}}>0$ is the continuity constant of $\gamma_{C}$. As $\left(\mu_{\bar{H} \bar{q}}^{*}\right)^{+} \in G^{*}$ and $\max \left\{\zeta_{\bar{h} \bar{p}}^{*}, \psi-\right.$ $v\} \in K_{v}$, Theorem 2.7 yields the assertion. 


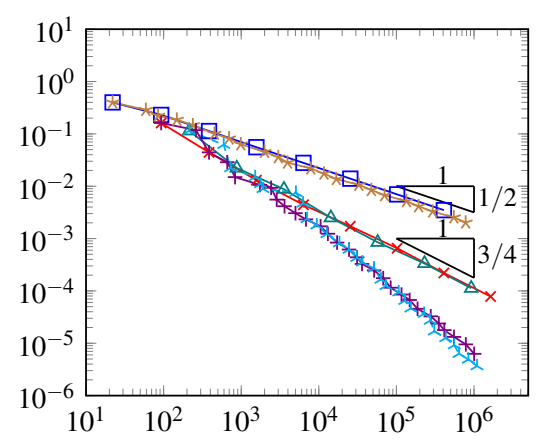

(A) $\|u-v\|_{H^{1}(\Omega)}$

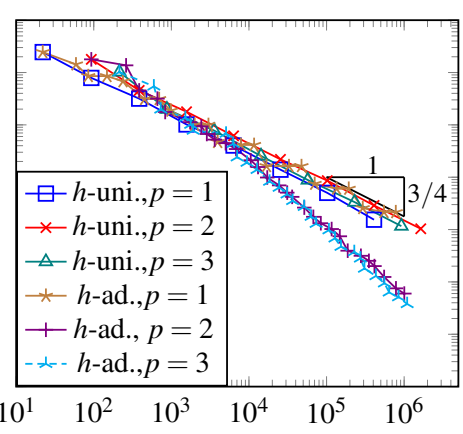

(B) $\chi\left(w_{h p}\right)$

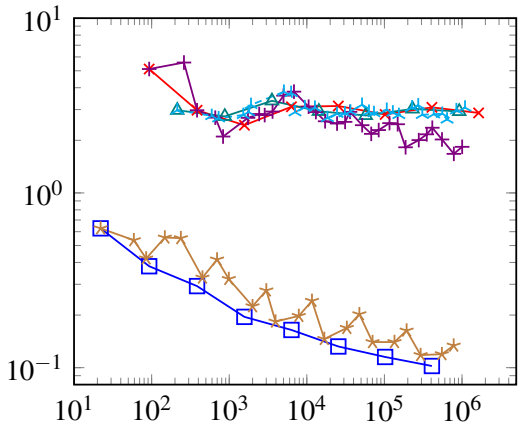

(C) Efficiency index

FIGURE 1. Discretization error and efficiency index with the degrees of freedom at the $x$-axis. Standard cut-off functions are used to steer adaptive schemes.

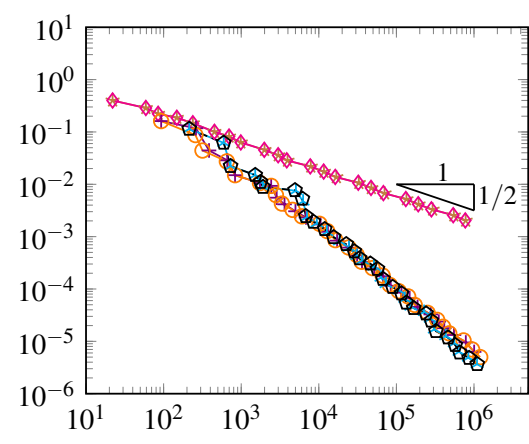

(A) $\|u-v\|_{H^{1}(\Omega)}$

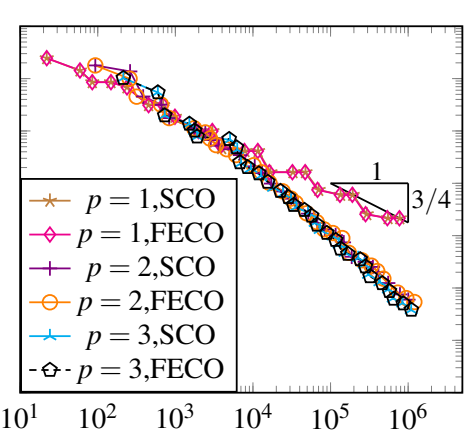

(B) $\chi\left(w_{h p}\right)$

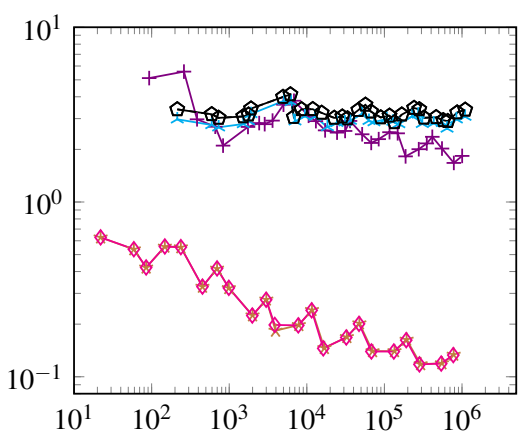

(C) Efficiency index

FIGURE 2. Discretization error and efficiency index with the degrees of freedom at the $x$-axis. Standard cut-off functions (SCO) and finite element cut-off functions (FECO) are used to steer adaptive schemes.

\section{NUMERICAL RESULTS}

In this section we report some numerical experiments regarding the a posteriori error estimates of the previous sections, in particular on the effect of the standard cut-off functions $\kappa^{+}$ and $(v-\psi)^{+}$from (3.10) and the finite element cut-off functions $\left(\mu_{\bar{H} \bar{q}}^{*}\right)^{+}$and $\max \left\{\zeta_{\bar{h} \bar{p}}^{*}, \psi-v\right\}$ proposed in Theorem 3.1. For this purpose, we consider the obstacle problem as introduced in Section 3.2 with $\Omega$ as the disc of radius 1.5 and midpoint 0 . We set $\Gamma_{D}:=\partial \Omega, f:=-2$ and $\psi:=-\left(1.5^{2}-\log \left(1.5^{2}\right)-1\right) / 2$ such that the solution $u$ of the obstacle problem becomes

$$
u(x, y)= \begin{cases}\psi, & x^{2}+y^{2} \leq 1 \\ \frac{x^{2}+y^{2}-\log \left(x^{2}+y^{2}\right)-1}{2}+\psi, & \text { otherwise }\end{cases}
$$

with

$$
\lambda(x, y)= \begin{cases}2, & x^{2}+y^{2} \leq 1 \\ 0, & \text { otherwise }\end{cases}
$$




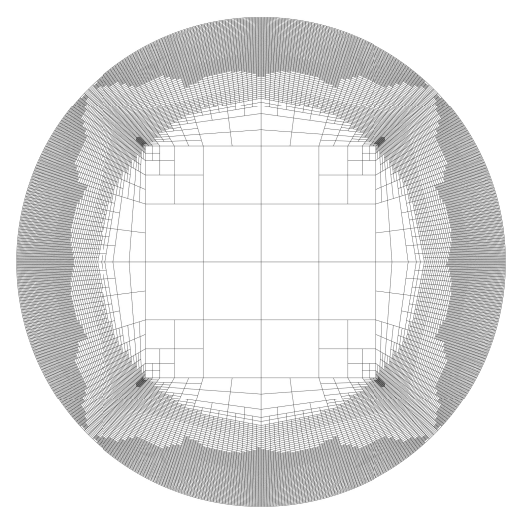

(A) Mesh nr. 18 for $p=1$

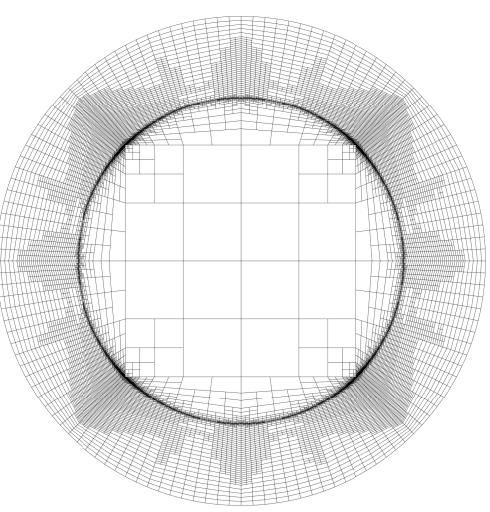

(B) Mesh nr. 25 for $p=2$

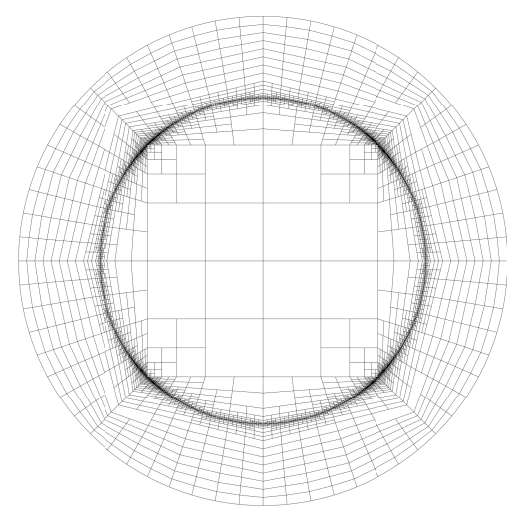

(C) Mesh nr. 25 for $p=3$

FIGURE 3. $h$-adaptively generated meshes

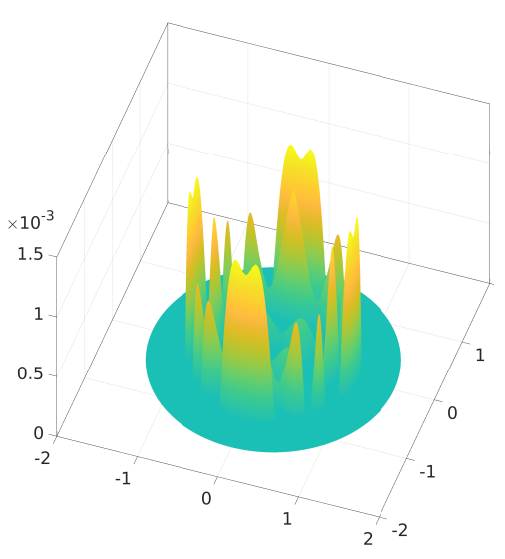

(A) $\max \{0, \psi-v\}$

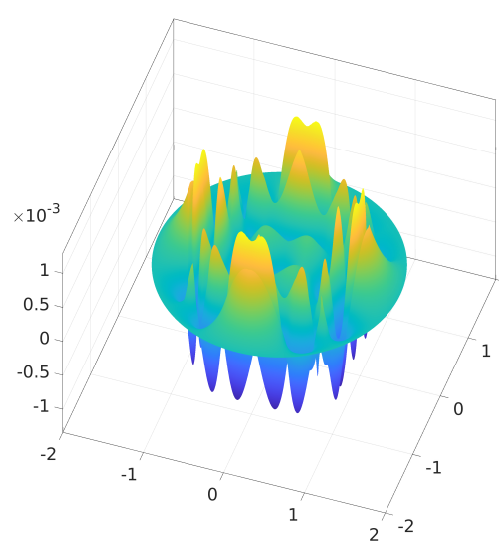

(B) $\max \left\{\zeta_{h p}^{*}, \psi-v\right\}$

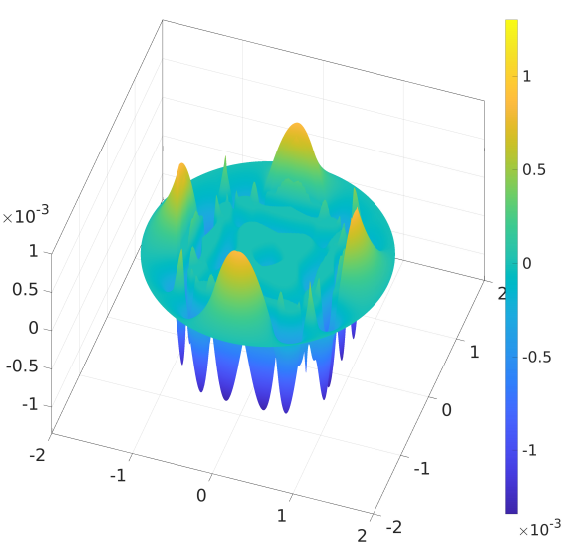

(C) Difference of cut-off functions

FIGURE 4. Cut-off functions for uniform $h$-version with $p=3$ and 80 elements

Note that $u$ is at most in $H^{5 / 2}(\Omega)$ and that the free boundary, a circle of radius 1 and center in 0 , is the location of the singularity. Thus the order of convergence resulting from finite element discretizations with uniform refinements is bounded by 0.75 with respect to the degrees of freedom and also adaptive refinements with isotropic divisions of the mesh elements cannot exhibit arbitrarily large convergence rates.

For the decomposition of the domain $\Omega$ we use quadrilaterals with straight and curved edges, i.e. mesh elements $T$ with $\partial T \cap \partial \Omega=\emptyset$ result from bilinear maps with translation $F_{T}:(0,1)^{2} \rightarrow$ $T$, whereas the maps $F_{T}$ are polynomials of degree $r$ in each direction for the remaining elements touching the boundary. This slightly differs from the decomposition $\Omega$ with parallelograms and affine maps $F_{T}$ as introduced in Section 3.1, which is needed for the reliability and efficiency results of the a posteriori error estimator $\hat{\eta}$ given in (3.7). But, this is not essential for the evaluation of the error estimates. The domain approximation errors $\Omega_{h}^{-}:=\left|\Omega \backslash \Omega_{h}\right|$ and $\Omega_{h}^{+}:=$ $\left|\Omega_{h} \backslash \Omega\right|$ with $\Omega_{h}:=\bigcup_{T \in \mathscr{T}_{h}} \bar{T}$ behave like $\mathscr{O}\left(h^{2 r}\right)$ with $\Omega_{h}^{+}$almost always being zero if the transformation mappings for the curved elements are based on nodal interpolation in some 


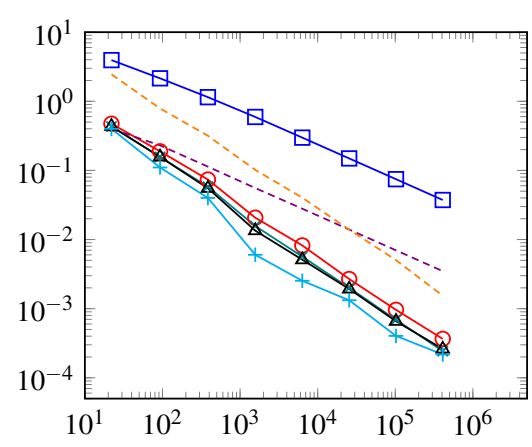

(A) $h$-uniform, $p=1$

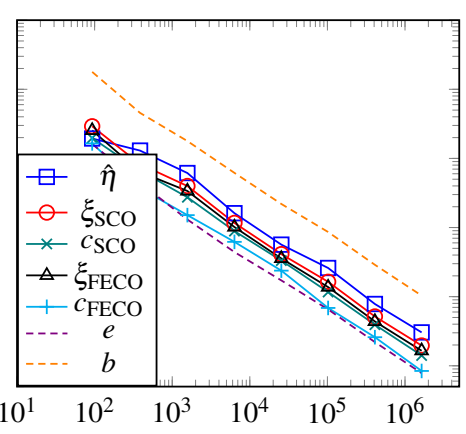

(B) $h$-uniform, $p=2$

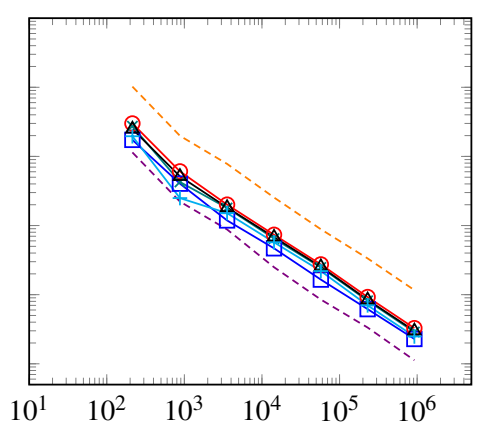

(C) $h$-uniform, $p=3$

FIGURE 5. Error contributions vs. degrees of freedom, when uniform refinements are applied.

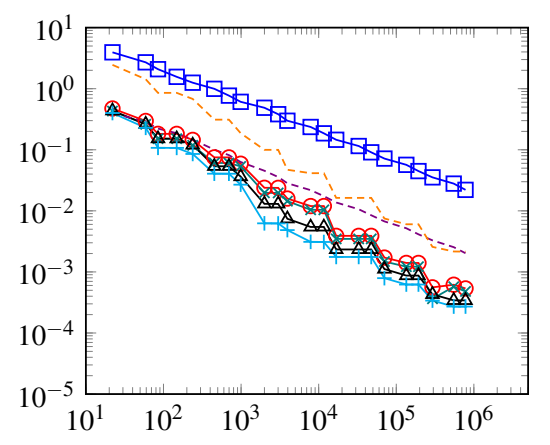

(A) $p=1$

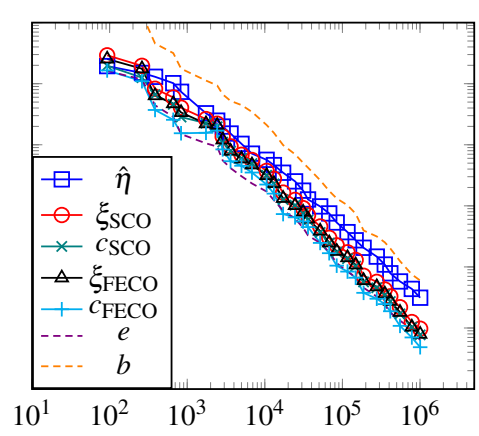

(B) $p=2$

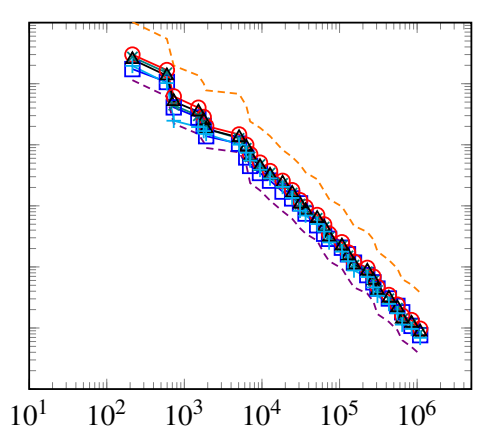

(C) $p=3$

FIGURE 6. Error contributions vs. degrees of freedom, when $h$-adaptive refinements with the standard cut-off functions are applied.

Gauss-Lobatto points. We set $r:=6$ in the experiments. For the initial mesh consisting of 5 elements we already have $\Omega_{h_{0}}^{-} \approx 3 \cdot 10^{-10}, \Omega_{h_{0} / 2}^{-} \approx 5 \cdot 10^{-14}$ and $\Omega_{h}^{+}=0$, so that we can neglect the domain approximation errors.

In our experiments we choose $(v, \kappa)$ as the solution of the mixed problem (3.5) with

$$
\Lambda_{H q}:=\left\{\mu_{H q} \in W_{H q} \mid \forall T \in \mathscr{T}_{H}, \forall x \in \mathscr{G}_{T}: \mu_{H q}(x) \geq 0\right\}
$$

where $\mathscr{T}_{H}$ is a further decomposition of $\Omega$ and $\mathscr{G}_{T}$ a set of transformed Gauss points. To satisfy the discrete inf-sup condition we reduce the polynomial degree for $\kappa$ by one compared to $v$ and the mesh for $v$ is obtained by one time uniformly refining the mesh for $\kappa$, i.e. we take $q:=p-1$ and $h:=H / 2$, see [35]. As $\lambda \in L^{2}(\Omega)$ (due to the regularity of $u$ ) we can compute lower and upper bounds for $\|\lambda-\kappa\|_{H_{D}^{-1}(\Omega)}$ by using the Riesz representation theorem yet with the discrete variational equation: find $w_{h p} \in Z_{h p} \subset H_{D}^{1}(\Omega)$ such that

$$
\left(\nabla w_{h p}, \nabla z_{h p}\right)_{L^{2}(\Omega)}=\left(\lambda-\kappa, z_{h p}\right)_{L^{2}(\Omega)}
$$


for all $z_{h p} \in Z_{h p}$. We estimate

$$
\beta\left\|w_{h p}\right\|_{H^{1}(\Omega)} \leq\|\lambda-\kappa\|_{H_{D}^{-1}(\Omega)}=\sup _{z \in H_{D}^{1}(\Omega) \backslash\{0\}} \frac{\langle\lambda-\kappa, z\rangle}{\|z\|_{H^{1}(\Omega)}} \leq \check{c} \chi\left(w_{h p}\right)
$$

with $\chi\left(w_{h p}\right):=\left\|w_{h p}\right\|_{H^{1}(\Omega)}+\|h \cdot(\lambda-\kappa)\|_{L^{2}(\Omega)}$ and a constant $\check{c}>0$, see [24] for more details on the estimation given by the last inequality. Thus, it is justified to take $\chi\left(w_{h p}\right)$ instead of $\|\lambda-\kappa\|_{H_{D}^{-1}(\Omega)}$ when, for instance, convergence rates are discussed. Note that the only challenge in the evaluation of $\chi\left(w_{h p}\right)$ is the quadrature due to the (potential) non-smoothness of $\lambda$. One may use an adaptive quadrature rule or as in our case an overkill Gauss quadrature rule with e.g. $p+15$ points per dimension. As $\lambda$ is essentially a Heaviside function the Gauss quadrature converges towards the exact integral value with some algebraic (but small) convergence rates.

Figure 1 and Figure 2 show the reduction of the error for different discretization methods, where the error estimates from (3.11) and Theorem 3.1 with $\varepsilon:=1$ are applied to steer the adaptive schemes. We consider discretizations with uniform $h$-refinements and $p=1,2,3$ and corresponding $h$-adaptive refinements based on Dörfler marking with bulk parameter $\theta=0.5$. In particular, Figure 2 shows that estimates (3.11) with the standard cut-off functions $\kappa^{+}$and $(v-\psi)^{+}$and the estimates as stated in Theorem 3.1 with the finite element cut-off functions $\left(\mu_{\bar{H} \bar{q}}^{*}\right)^{+}$and $\max \left\{\zeta_{\bar{h} \bar{p}}^{*}, \psi-v\right\}$ do not siginificantly differ. To compute the finite element cutoff functions, we use the same discretization spaces as for $v$ and $\kappa$, i.e. $\bar{H}=H, \bar{q}=q, \bar{h}=h$ and $\bar{p}=p$ and apply the slight simplification of the discretization systems (3.15) and (3.16) as proposed in Remark 3.2. As expected the experimental order of convergence (eoc) with respect to the degrees of freedom for the uniform $h$-versions are $0.5,0.75$ and 0.75 for $p=$ $1,2,3$, respectively. It is noteworthy that the error in the Lagrange multiplier $\lambda$ converges at an increased rate of 0.75 for $p=1$ and both uniform and adaptive meshes. The eocs for the adaptive refinements are optimal 0.5 for $p=1$, increased 1.2 for $p=2$ which should tend to one, and 1.38 for $p=3$. All adaptive schemes identify the contact zone in which the solution is constant and thus no mesh refinements need to be carried out, see Figure 3. We observe that the higher the polynomial degree $p$ the stronger the concentration of the mesh refinements to the free boundary. In Figure 4, a standard cut-off function, the corresponding finite element cut-off function and their difference are depicted. Both graphs are very similar with the finite element cut-off function appearing to be less angular and sometimes also being negative where $v$ is not in contact with the obstacle $\psi$.

As in the previous works $[24,25,26,27]$ we observe that the standard cut-off functions yield reliable a posteriori error estimates in theory, which also seems to be efficient in the numerical experiments. Surprisingly, the finite element cut-off functions have only small positive effect on the efficiency index (error divided by error estimate), see Figure 2, and almost no effect on the adaptive schemes in comparison to the use of the standard cut-off functions. The seemingly worsening efficiency constant for the discretization schemes with $p=1$ is due to the higher-order converging error in the dual variable $\lambda$ which for coarse and moderate meshes is the dominant contribution in the combined $H^{1}$ - and $H^{-1}$-error, see Figure 5. Looking at the individual contributions of the a posteriori error estimates with $\hat{\eta}$ as the residual error estimator 
of the auxiliary problem (3.6) and

$$
\begin{aligned}
\xi_{\mathrm{SCO}} & :=\left(\Xi_{\varepsilon}\left(\kappa^{+},(v-\psi)^{+}\right)\right)^{1 / 2} \\
c_{\mathrm{SCO}} & :=\left(\left(\kappa^{+}, v-\psi\right)_{L^{2}(\Omega)}+\left(\kappa,(v-\psi)^{+}\right)_{L^{2}(\Omega)}\right)^{1 / 2} \\
\xi_{\mathrm{FECO}} & :=\left(\Xi_{\varepsilon}\left(\left(\mu_{H q}^{*}\right)^{+}, \max \left\{\zeta_{\bar{h} \bar{p}}^{*}, \psi-v\right\}\right)^{1 / 2}\right. \\
c_{\mathrm{FECO}} & :=\left(\left(\left(\mu_{H q}^{*}\right)^{+}, v-\psi\right)_{L^{2}(\Omega)}+\left(\kappa, \max \left\{\zeta_{h p}^{*}, \psi-v\right\}\right)_{L^{2}(\Omega)}\right)^{1 / 2} \\
e & :=\|u-v\|_{H^{1}(\Omega)} \\
b & :=\chi\left(w_{h p}\right)
\end{aligned}
$$

we observe that $\hat{\eta}$ is by far the dominant contribution, see Figures 5 and 6 . We also observe that the complementary contributions are always positive in our experiments. Thus, in the figures we plot the square root $c_{\mathrm{SCO}}$ and $c_{\mathrm{FECO}}$ of these contributions. The quotient $\left(\xi_{\mathrm{SCO}} / \xi_{\mathrm{FE}}\right)^{1 / 2}$ is about $1.4,1.2,1.1$ for $p=1,2,3$, respectively.

\section{APPENDIX A. THE POSITIVE PART AND THE MINIMIZATION OF QUADRATIC FUNCTIONALS}

Let $H$ be a real Hilbert space and $C \subset H$ be a closed and convex cone with $0 \in C$. We define the positive part $v_{C}^{+} \in C$ for $v \in H$ as the unique projection of $v$ onto $C$, i.e.

$$
\left(v_{C}^{+}-v, w-v_{C}^{+}\right)_{H} \geq 0
$$

for all $w \in C$, where $(\cdot, \cdot)_{H}$ denotes the inner product of $H$. The negative part is defined by $v_{C}^{-}:=v-v_{C}^{+}$. It can easily be verified that

$$
\left(v_{C}^{+}, v_{C}^{-}\right)_{H}=0, \quad\left(w, v_{C}^{-}\right)_{H} \leq 0
$$

for arbitrary $v \in H$ and $w \in C$ and that

$$
\left\|v_{C}^{+}-\tilde{v}_{C}^{+}\right\|_{H} \leq\|v-\tilde{v}\|_{H}
$$

for all $v, \tilde{v} \in H$.

Lemma A.1. Let $\rho, \sigma, \tau \in Y$ and $F(v):=\|v-\rho\|_{H}^{2}+(v, \sigma)_{H}$. With $v^{*}:=\tau+\left(\rho-\frac{1}{2} \sigma-\tau\right)_{C}^{+}$it holds

$$
F\left(v^{*}\right)=\min _{v-\tau \in C} F(v)
$$

Proof. Let $v \in Y$ with $v-\tau \in C$. Obviously, we have $v^{*}-\tau \in C$. It holds

$$
\begin{aligned}
F(v)= & \|v-\tau-(\rho-\tau)\|_{H}^{2}+(v-\tau, \sigma)_{H}+(\tau, \sigma)_{H} \\
= & (v-\tau, v-\tau)_{H}-2\left(v-\tau,\left(\rho-\frac{1}{2} \sigma-\tau\right)_{C}^{+}\right)_{H}-2\left(v-\tau,\left(\rho-\frac{1}{2} \sigma-\tau\right)_{C}^{-}\right)_{H} \\
& +\|\rho-\tau\|_{H}^{2}+(\tau, \sigma)_{H} \\
= & \left\|v-v^{*}\right\|_{H}^{2}-2\left(v-\tau,\left(\rho-\frac{1}{2} \sigma-\tau\right)_{C}^{-}\right)_{H}+\|\rho-\tau\|_{H}^{2}+(\tau, \sigma)_{H}-\left\|\left(\rho-\frac{1}{2} \sigma-\tau\right)_{C}^{+}\right\|_{H}^{2} .
\end{aligned}
$$

From (A.1) we conclude

$$
F(v) \geq\|\rho-\tau\|_{H}^{2}+(\tau, \sigma)_{H}-\left\|\left(\rho-\tau-\frac{1}{2} \sigma\right)_{C}^{+}\right\|_{H}^{2},
$$


where equality holds if and only if $v=v^{*}$.

\section{Acknowledgments}

The second author gratefully acknowledges the support by the Deutsche Forschungsgemeinschaft in the Priority Programme 1748 'Reliable simulation techniques in solid mechanics. Development of non-standard discretization methods, mechanical and mathematical analysis' under the project 'High-order immersed-boundary methods in solid mechanics for structures generated by additive processes', Grant SCHR 1244/4-2.

\section{REFERENCES}

[1] G. Duvaut, J.-L. Lions, Inequalities in mechanics and physics, Springer-Verlag, Berlin - New York, 1976. Translated from the French by C. W. John, Grundlehren der Mathematischen Wissenschaften, 219.

[2] R. Glowinski, Numerical methods for nonlinear variational problems, Springer Series in Computational Physics, Springer-Verlag, New York, 1984.

[3] I. Hlaváček, J. Haslinger, J. Nečas, and J. Lovíšek, Solution of variational inequalities in mechanics, Applied Mathematical Sciences, vol. 66, Springer-Verlag, New York, 1988.

[4] N. Kikuchi, J. T. Oden, Contact problems in elasticity: a study of variational inequalities and finite element methods, SIAM Studies in Applied Mathematics, vol.8, Society for Industrial and Applied Mathematics (SIAM), Philadelphia, PA, 1988. doi: 10.1137/1.9781611970845.

[5] W. Han, B.D. Reddy, Plasticity, Interdisciplinary Applied Mathematics, vol. 9, Springer, New York, second edition, 2013. doi: 10.1007/978-1-4614-5940-8.

[6] F. Black, M. Scholes, The pricing of options and corporate liabilities, J. Polit. Econ. 81 (1973), 637-654.

[7] M. Ainsworth, J.T. Oden, A posteriori error estimation in finite element analysis, Pure and Applied Mathematics (New York), John Wiley \& Sons, New York, 2000. doi: 10.1002/9781118032824

[8] R. Verfürth, A posteriori error estimation techniques for finite element methods, Numerical Mathematics and Scientific Computation, Oxford University Press, Oxford, 2013.

[9] S. Bartels, C. Carstensen, Averaging techniques yield reliable a posteriori finite element error control for obstacle problems, Numer. Math. 99 (2004), 225-249.

[10] C. Carstensen, K. Köhler, Efficient discrete Lagrange multipliers in three first-order finite element discretizations for the a posteriori error control in an obstacle problem, SIAM J. Numer. Anal. 55 (2017), 349-375.

[11] Z. Chen, R.H. Nochetto, Residual type a posteriori error estimates for elliptic obstacle problems, Numer. Math. 84 (2000), 527-548.

[12] P. Hild, S. Nicaise, A posteriori error estimations of residual type for Signorini's problem, Numer. Math. 101 (2005), 523-549.

[13] P. Hild, S. Nicaise, Residual a posteriori error estimators for contact problems in elasticity, ESAIM: Math. Model. Numer. Anal. 41 (2007), 897-923.

[14] R. Krause, A. Veeser, M. Walloth, An efficient and reliable residual-type a posteriori error estimator for the Signorini problem, Numer. Math. 130 (2015), 151-197.

[15] A. Veeser, Efficient and reliable a posteriori error estimators for elliptic obstacle problems, SIAM J. Numer. Anal. 39 (2001), 146-167.

[16] A. Weiss, B.I. Wohlmuth, A posteriori error estimator for obstacle problems, SIAM J. Sci. Comput. 32 (2010), 2627-2658.

[17] D. Braess, R.H.W. Hoppe, J. Schröder, A posteriori estimators for obstacle problems by the hypercircle method, Comput. Vis. Sci. 11 (2008), 351-362.

[18] H. Blum, A. Schröder, F.T. Suttmeier, A posteriori estimates for FE-solutions of variational inequalities. In Numerical mathematics and advanced applications, pp. 669-680, Springer, Milan, 2003.

[19] A. Rademacher, A. Schröder, Dual weighted residual error control for frictional contact problems, Comput. Methods Appl. Math. 15 (2015), 391-413. 
[20] A. Schröder, A. Rademacher, Goal-oriented error control in adaptive mixed FEM for Signorini's problem, Comput. Methods Appl. Mech. Eng. 200 (2011), 345-355.

[21] D. Braess, A posteriori error estimators for obstacle problems-another look, Numer. Math. 101 (2005), 415-421.

[22] L. Banz, Bishnu P. Lamichhane, E.P. Stephan, Higher order mixed FEM for the obstacle problem of the p-Laplace equation using biorthogonal systems, Comput. Methods Appl. Math. 19 (2019), 169-188.

[23] L. Banz, B.P. Lamichhane, E.P. Stephan, Higher order FEM for the obstacle problem of the p-Laplacian - a variational inequality approach. Comput. Math. Appl. 76 (2018), 1639-1660.

[24] L. Banz, A. Schröder, Biorthogonal basis functions in hp-adaptive FEM for elliptic obstacle problems, Comput. Math. Appl. 70 (2015), 1721-1742.

[25] A. Schröder, A posteriori error estimates of higher-order finite elements for frictional contact problems, Comput. Methods Appl. Mech. Eng. 249/252 (2012), 151-157.

[26] A. Schröder, Error control in h- and hp-adaptive FEM for Signorini's problem, J. Numer. Math. 17 (2009), 299-318.

[27] M. Bürg, A. Schröder, A posteriori error control of hp-finite elements for variational inequalities of the first and second kind, Comput. Math. Appl. 70 (2015), 2783-2802.

[28] A. Schröder, A posteriori error estimation in mixed finite element methods for Signorini's problem, In Numerical Mathematics and Advanced Applications 2009. Proceedings of ENUMATH 2009, the 8th European Conference on Numerical Mathematics and Advanced Applications, Uppsala, Sweden, June 29-July 3, 2009, pp. 801-808, Springer, Berlin, 2010.

[29] L. Banz, H. Gimperlein, A. Issaoui, E.P. Stephan, Stabilized mixed hp-BEM for frictional contact problems in linear elasticity, Numer. Math. 135 (2017), 217-263.

[30] L. Banz, E.P. Stephan, On hp-adaptive BEM for frictional contact problems in linear elasticity, Comput. Math. Appl. 69 (2015), 559-581.

[31] L. Banz, E.P. Stephan, Comparison of mixed hp-BEM (stabilized and non-stabilized) for frictional contact problems, J. Comput. Appl. Math. 295 (2016), 92-102.

[32] N. Ovcharova, L, Banzm Coupling regularization and adaptive hp-BEM for the solution of a delamination problem, Numer. Math. 137 (2017), 303-337.

[33] J. M. Melenk, B. I. Wohlmuth, On residual-based a posteriori error estimation in hp-FEM, Adv. Comput. Math. 15 (2001), 311-331.

[34] D. Kinderlehrer, G. Stampacchia, An introduction to variational inequalities and their applications, Classics in Applied Mathematics, vol. 31, Society for Industrial and Applied Mathematics (SIAM), Philadelphia, PA, 2000. doi: 10.1137/1.9780898719451.

[35] A. Schröder, Mixed finite element methods of higher-order for model contact problems, SIAM J. Numer. Anal. 49 (2011), 2323-2339.

[36] A. Krebs, E.P. Stephan, A p-version finite element method for nonlinear elliptic variational inequalities in 2D, Numer. Math. 105 (2007), 457-480.

[37] A. Schröder, H. Kleemann, H. Blum. Mixed finite element methods for two-body contact problems, J. Comput. Appl. Math. 283 (2015), 58-70. 